TRANSACTIONS OF THE

AMERICAN MATHEMATICAL SOCIETY

Volume 357, Number 12, Pages 4839-4866

S 0002-9947(05)04005-5

Article electronically published on July 19, 2005

\title{
COMPLETE ANALYTIC EQUIVALENCE RELATIONS
}

\author{
ALAIN LOUVEAU AND CHRISTIAN ROSENDAL
}

\begin{abstract}
We prove that various concrete analytic equivalence relations arising in model theory or analysis are complete, i.e. maximum in the Borel reducibility ordering. The proofs use some general results concerning the wider class of analytic quasi-orders.
\end{abstract}

\section{INTRODUCTION}

This paper is part of the general theory of analytic equivalence relations, i.e. structures $(X, E)$, where $X$ is a Polish space (or more generally a standard Borel space, for the notions only depend on the Borel structure of $X$ ) and $E$ is an equivalence relation on $X$ which is $\boldsymbol{\Sigma}_{1}^{1}$ (analytic) as a subset of $X^{2}$. This theory is organized by a quasi-order, $\leq_{B}$, called Borel reducibility: If $E$ and $F$ with respective domains $X$ and $Y$ are $\boldsymbol{\Sigma}_{1}^{1}$ equivalence relations, a Borel reduction from $E$ to $F$ is a Borel map $f: X \rightarrow Y$ which satisfies, for all $x, y$ in $X$,

$$
x E y \Longleftrightarrow f(x) F f(y)
$$

and $E \leq_{B} F$ if there exists a Borel reduction from $E$ to $F$. The associated equivalence relation $\sim_{B}$, defined by

$$
E \sim_{B} F \Longleftrightarrow E \leq_{B} F \text { and } F \leq_{B} E,
$$

is called Borel bi-reducibility.

The intuitive meaning of the order of Borel reducibility is the following: If we view $X$ as a set of mathematical objects, which we are interested in up to $E$ equivalence, the classification problem for $(X, E)$ consists of finding some (concrete, or nicely definable) set $I$ of invariants, together with some (concrete, or nicely definable) assignment $\varphi: X \rightarrow I$, which completely classifies elements in $X$ up to $E$-equivalence, i.e. satisfies $x E y \Longleftrightarrow \varphi(x)=\varphi(y)$. As any solution to the classification problem for $(Y, F)$ gives, by composing with a Borel reduction from $E$ to $F$, a solution to the classification problem for $(X, E)$, Borel reducibility intuitively corresponds to a comparison of the complexity of classification problems - at least when the sets are Polish and the equivalence relations are $\boldsymbol{\Sigma}_{1}^{1}$, which is very often the case.

It is now well established that the ordering $\leq_{B}$ is extremely complicated (see [1,22]), and a good part of the recent developments of the theory has been to isolate some $\left(\sim_{B}\right.$-degrees of $)$ specific equivalence relations which can be used as milestones to get information about the complexity of various natural classification problems.

Received by the editors May 14, 2003.

2000 Mathematics Subject Classification. Primary 03E15.

Key words and phrases. Definable equivalence relations, descriptive set theory. 
It so happens that in most cases, these milestones are the $\leq_{B}$-maximum elements in various natural classes of $\boldsymbol{\Sigma}_{1}^{1}$ equivalence relations (a fact which is very useful to get upper bounds to the complexity of natural problems).

The purpose of this paper is to study one of these milestones, namely the degree consisting of all $\leq_{B^{-}}$maximum $\boldsymbol{\Sigma}_{1}^{1}$ equivalence relations. Elements in this $\sim_{B^{-}}$ degree, i.e. $\boldsymbol{\Sigma}_{1}^{1}$ equivalence relations which Borel reduce all other $\boldsymbol{\Sigma}_{1}^{1}$ equivalence relations, will be called complete $\boldsymbol{\Sigma}_{1}^{1}$ equivalence relations (they are sometimes called universal). Our main goal is to develop a "completeness" method for them, i.e. tools for proving that a given pair $(X, E)$ is complete, and then to apply these tools to specific natural classification problems.

In order to make what we plan to do more precise, let us look first at a very similar - but much simpler - situation, in dimension 1 . The objects there are pairs $(X, A)$, where $A$ is now a $\boldsymbol{\Sigma}_{1}^{1}$ subset of the Polish space $X$, and Borel reducibility of $A$ to $B$ now corresponds to the existence of a Borel map $f$ between the ambient spaces with $A=f^{-1}(B)$. Again maximum elements are called complete $\boldsymbol{\Sigma}_{1}^{1}$ sets, and their existence follows from the existence of universal $\boldsymbol{\Sigma}_{1}^{1}$ sets (which are clearly necessarily complete).

In dimension 1, the ordering of Borel reducibility is not very interesting, for apart from the trivial cases of $\{\emptyset\}$ and its dual class (consisting of all $(X, X), X$ Polish), all Borel sets fall in one degree. Moreover the determinacy of $\boldsymbol{\Sigma}_{1}^{1}$ games implies that every $\boldsymbol{\Sigma}_{1}^{1}$ non-Borel set is complete. However, the so-called "completeness" method, which goes back to the beginning of Descriptive Set Theory, has proved to be very fruitful: in order to prove that a given $\boldsymbol{\Sigma}_{1}^{1}$ set is not Borel, one just Borel reduces to it some other $\boldsymbol{\Sigma}_{1}^{1}$ set, already known to be complete. The method has the advantage of being cumulative, even if one very often uses as test sets a few very specific complete sets, which are combinatorially easy to deal with, like the set $N W F$ of ill-founded countable trees, or the set $N W O$ of non-well-ordered linear orderings. Of course, one must first establish the completeness of these test sets, which is classically done by deriving it from a representation, or "normal form", result for $\boldsymbol{\Sigma}_{1}^{1}$ sets.

In this paper, we will follow the same patterns, but in dimension 2, and for $\boldsymbol{\Sigma}_{1}^{1}$ equivalence relations (and, as we will see shortly below, for the larger class of $\boldsymbol{\Sigma}_{1}^{1}$ quasi-orders).

As in dimension 1, the existence of complete objects follows easily from the existence of universal ones. Although the reducibility ordering is now very complicated, so that in particular there are lots of non-Borel but also non-complete $\boldsymbol{\Sigma}_{1}^{1}$ equivalence relations, one can still start a completeness method, by first defining a "combinatorially simple" object, then proving a representation result which ensures its completeness, and in a third step using it to prove the completeness of various natural classification problems in Logic and Analysis.

This is the program we will follow, with one important change: For reasons which are not totally clear to us, but might be intrinsic, the preceding program is easier to develop for the class of $\boldsymbol{\Sigma}_{1}^{1}$ quasi-orders (or partial pre-orders, i.e. binary relations which are reflexive and transitive, but not necessarily symmetric). By symmetrization, we will recover all desired results for the class of $\boldsymbol{\Sigma}_{1}^{1}$ equivalence relations, which is our main concern. So in some sense we are performing an even broader task. However, from a different viewpoint, this can be viewed as a serious limitation to our method, for the "combinatorial simplicity" is lost in the process 
of symmetrizing. In particular, we do not know how to prove that a given $\boldsymbol{\Sigma}_{1}^{1}$ equivalence relation is complete, unless it naturally comes with a $\boldsymbol{\Sigma}_{1}^{1}$ quasi-order which we can prove to be complete.

The paper is organized as follows: In section 1, we collect some facts about the reducibility order $\leq_{B}$, including the existence of $\leq_{B}$-maximum elements for various classes. We also very briefly describe the milestones among $\Sigma_{1}^{1}$ equivalence relations that will be useful in the paper.

In section 2 , we define our basic complete $\boldsymbol{\Sigma}_{1}^{1}$ quasi-order, $\leq_{\max }$, and prove a representation result which implies its completeness. We also use it to briefly discuss the case of Borel quasi-orders and Borel equivalence relations.

The last three sections contain applications of our completeness method to various classification problems in Model Theory (section 3), in Analysis (section 4) and to Polish groups and monoids (section 5).

For example, we prove in section 3 that bi-embeddability between countable partial orders, and bi-embeddability between countable combinatorial trees, are complete $\boldsymbol{\Sigma}_{1}^{1}$ equivalence relations. In section 4 , we prove the same result for continuous bi-embeddability between compact metrizable spaces, isometric bi-embeddability between Polish metric spaces, and linear isometric bi-embeddability between separable Banach spaces. Finally we see in section 5 that there is a Polish semigroup (in fact a monoid) acting on a Polish space inducing a complete $\boldsymbol{\Sigma}_{1}^{1}$ quasi-order.

The main results in this paper were announced in a Note aux Comptes Rendus de l'Académie des Sciences, 21.

\section{The Borel ReducibiLity ORDERING}

We first define the order $\leq_{B}$ in a very general form.

Definition 1.1. Let $A_{1}$ and $A_{2}$ be two binary relations on Polish spaces $X_{1}$ and $X_{2}$, respectively.

A map $f$ from $X_{1}$ to $X_{2}$ is a reduction of $A_{1}$ to $A_{2}$ if for all $x, y$ in $X_{1}$,

$$
(x, y) \in A_{1} \Longleftrightarrow(f(x), f(y)) \in A_{2} .
$$

We say that $A_{1}$ is Borel reducible to $A_{2}$, or $A_{2}$ Borel reduces $A_{1}$, in symbols $A_{1} \leq_{B} A_{2}$, if there is a Borel reduction $f$ from $A_{1}$ to $A_{2}$. If moreover the reduction $f$ can be found one-to-one, we say that $A_{1}$ Borel embeds into $A_{2}\left(A_{1} \sqsubseteq_{B} A_{2}\right)$.

Finally, we denote by $\sim_{B}$ the associated equivalence relation of Borel bireducibility, defined by

$$
A_{1} \sim_{B} A_{2} \Longleftrightarrow A_{1} \leq_{B} A_{2} \wedge A_{2} \leq_{B} A_{1} .
$$

As we said in the Introduction, Borel reducibility has been studied mainly in the context of $\boldsymbol{\Sigma}_{1}^{1}$ equivalence relations. There, because of the reflexivity condition, the domain of the relation is important, and Polish spaces are often replaced by standard Borel spaces - a harmless change, as a standard Borel space is just a Polish space of which only the Borel structure is considered - and sometimes are replaced by arbitrary subsets of Polish spaces. We will not consider this second generalization here. Another possible generalization would be to consider more general structures with Polish domain, and relations and functions of arbitrary arity.

First, let us note some simple features of $\leq_{B}$. It is clearly reflexive and transitive, i.e. a quasi-order. Also, the Borelness condition imposed on the reductions 
implies that for most descriptive classes $\Gamma$, and in particular for the class $\boldsymbol{\Sigma}_{1}^{1}$ we are interested in, binary relations in $\Gamma$ are downward closed under $\leq_{B}$, or equivalently relations in $\Gamma$ can only reduce relations in $\Gamma$. But there are also other limitations, of a more algebraic type, due to the special "square" form of the reductions: The properties of reflexivity, antireflexivity, symmetry and transitivity are all preserved downwards by reductions. So quasi-orders can only reduce quasi-orders, and equivalence relations can only reduce equivalence relations.

One can push these remarks a bit further. Let $R$ be a quasi-ordering. Denote by $R \backsim$ its dual (or reverse) ordering, i.e. $x R^{\curlyvee} y$ in the case $y R x$. Let $\equiv_{R}=R \cap R \backsim$ be the associated equivalence relation, and let $<_{R}=\left(R \backslash \equiv_{R}\right)$ be the associated strict order. Then if $R_{1}$ and $R_{2}$ are quasi-orders and $f$ is a reduction of $R_{1}$ to $R_{2}$, $f$ also reduces $R_{1}$ to $R_{2}$, $\equiv_{R_{1}}$ to $\equiv_{R_{2}}$, and $<_{R_{1}}$ to $<_{R_{2}}$. This explains why we will be able to get results about $\Sigma_{1}^{1}$ equivalence relations from similar results about $\Sigma_{1}^{1}$ quasi-orders.

Another nice feature of $\leq_{B}$ is that the particular ambient Polish space $X$ is irrelevant in the general theory, as any two uncountable Polish spaces are Borel isomorphic, and hence any $\sim_{B}$-degree has members in any given uncountable Polish space $X_{0}$.

Definition 1.2. Let $\mathcal{C}$ be a class of binary relations on Polish spaces. A relation $A$ is $\mathcal{C}$-complete, or complete in $\mathcal{C}$, if $A \in \mathcal{C}$ and $A$ Borel reduces all elements of $\mathcal{C}$, i.e. has maximum $\leq_{B}$-degree among elements of $\mathcal{C}$.

When the class $\mathcal{C}$ is clear from the context, we will just say that $A$ is complete. Most classes $\mathcal{C}$ that we will consider are downward closed under $\leq_{B}$, so that this abuse of terminology is harmless. E.g. a $\boldsymbol{\Sigma}_{1}^{1}$ equivalence relation can only be complete among $\boldsymbol{\Sigma}_{1}^{1}$ equivalence relations.

Proposition 1.3. The following classes admit complete elements:

- $\Sigma_{1}^{1}$ binary relations,

- $\boldsymbol{\Sigma}_{1}^{1}$ reflexive (resp. irreflexive) relations,

- $\boldsymbol{\Sigma}_{1}^{1}$ symmetric (resp. symmetric reflexive, symmetric irreflexive) relations,

- $\boldsymbol{\Sigma}_{1}^{1}$ quasi-orders,

- $\Sigma_{1}^{1}$ equivalence relations.

Proof. We use the existence of universal sets. Let $W_{0} \subseteq\left(2^{\omega}\right)^{3}$ be a $\boldsymbol{\Sigma}_{1}^{1}$ set universal for $\Sigma_{1}^{1}$ subsets of $2^{\omega} \times 2^{\omega}$. Define $W_{1}$ on $2^{\omega} \times 2^{\omega}$ by

$$
\left(\alpha_{1}, \alpha_{2}\right) W_{1}\left(\beta_{1}, \beta_{2}\right) \leftrightarrow \alpha_{1}=\beta_{1} \wedge\left(\alpha_{1}, \alpha_{2}, \beta_{2}\right) \in W_{0} .
$$

$W_{1}$ is a $\Sigma_{1}^{1}$ binary relation on $2^{\omega} \times 2^{\omega}$. To check that it is complete, it is enough to Borel reduce to it any binary relation $A$ on $2^{\omega}$, by the remark above. But if $\alpha$ is a $W_{0}$-code of $A$, the map $\beta \mapsto(\alpha, \beta)$ reduces $A$ to $W_{1}$.

The other cases are very similar. If $\Delta$ is equality on $2^{\omega} \times 2^{\omega}$, then $W_{1} \cup \Delta$ is complete for reflexive $\boldsymbol{\Sigma}_{1}^{1}$ relations, $W_{1} \backslash \Delta$ for irreflexive ones, and $W_{1} \cap W_{1}$, $\left(W_{1} \cap W_{1}^{\jmath}\right) \cup \Delta$ and $\left(W_{1} \cap W_{1}^{\jmath}\right) \backslash \Delta$ are complete for symmetric, symmetric reflexive, and symmetric irreflexive $\boldsymbol{\Sigma}_{1}^{1}$ relations, respectively.

The quasi-order $W_{2}$ generated by $W_{1}$ is also complete for $\boldsymbol{\Sigma}_{1}^{1}$ quasi-orders. First it is easily $\boldsymbol{\Sigma}_{1}^{1}$ (as $\boldsymbol{\Sigma}_{1}^{1}$ is closed under projections). Also, If $R$ is any $\boldsymbol{\Sigma}_{1}^{1}$ quasi-order on $2^{\omega}$, with $W_{0}$-code $\alpha$, one still has

$$
\beta_{1} R \beta_{2} \leftrightarrow\left(\alpha, \beta_{1}\right) W_{2}\left(\alpha, \beta_{2}\right)
$$


so that $W_{2}$ is indeed "universal" for $\Sigma_{1}^{1}$ quasi-orders, and the map $\beta \mapsto(\alpha, \beta)$ again reduces $R$ to $W_{2}$. The case of equivalence relations is similar, working now with the equivalence relation $W_{3}$ generated by $W_{1}$.

This very easy proof used only closure of $\boldsymbol{\Sigma}_{1}^{1}$ under Borel preimages, countable unions and projections, and hence works as well for many descriptive classes $\Gamma$, e.g. $\boldsymbol{\Sigma}_{n}^{1}$. It can also be adapted to the class $\mathbf{K}_{\sigma}$ (which is closed under countable unions and projections, but not Borel preimages).

Proposition 1.4. The classes of $\mathbf{K}_{\sigma}$ quasi-orders and $\mathbf{K}_{\sigma}$ equivalence relations (on compact Polish spaces) admit complete elements.

Proof. Starting from a $\mathbf{K}_{\sigma}$ subset of $2^{\omega} \times\left(2^{\omega} \times 2^{\omega}\right)$ universal for $\mathbf{K}_{\sigma}$ subsets of $2^{\omega} \times 2^{\omega}$, we get as before a $\mathbf{K}_{\sigma}$ quasi-order on $2^{\omega} \times 2^{\omega}$ which is "universal" for $\mathbf{K}_{\sigma}$ quasi-orders on $2^{\omega}$. So it is enough to check that any $\mathbf{K}_{\sigma}$ quasi-order $(X, R)$, with $X$ compact Polish, is Borel bi-reducible with one on $2^{\omega}$. But there is a continuous onto map $\pi: 2^{\omega} \rightarrow X$, with Borel selection $s: X \rightarrow 2^{\omega}$. Then $R^{\prime}=(\pi \otimes \pi)^{-1}(R)$ is a $\mathbf{K}_{\sigma}$ quasi-order on $2^{\omega}$, and $\pi$ and $s$ witness that $R \sim_{B} R^{\prime}$. The proof is similar for $\mathbf{K}_{\sigma}$ equivalence relations.

For classes with different closure properties, other techniques have to be used. In [11, Hjorth proves the existence of a complete (in fact "universal") $\Pi_{1}^{1}$ equivalence relation, using the norm and boundedness properties of this class. His proof extends to $\Pi_{1}^{1}$ quasi-orders as well. In the next section, we will see a different approach for compact quasi-orders, which also extends to closed quasi-orders (on Polish spaces) with some more work; see Louveau [19]. This other approach works also for $\mathbf{K}_{\sigma}$ and $\boldsymbol{\Sigma}_{1}^{1}$ quasi-orders, and will be instrumental for the results of section 2.

In the opposite direction, there are classes with no complete element. H. Friedmann proved this first for the class of Borel equivalence relations (see [29] and [17]), and it extends easily to Borel quasi-orders. The class of $\boldsymbol{\Pi}_{2}^{0}$ quasi-orders has no complete element (Louveau [18]) (but equality on $2^{\omega}$ is complete for $\boldsymbol{\Pi}_{2}^{0}$ equivalence relations). Also, the class of $\boldsymbol{\Sigma}_{1}^{1}$ strict orders has no complete element (Louveau [20]).

Proposition 1.5. Let $E$ be a $\Sigma_{1}^{1}$ equivalence relation on the Polish space $X$. Then $E$ is complete if and only if $E=\equiv_{R}$, for some complete $\boldsymbol{\Sigma}_{1}^{1}$ quasi-order $R$ on $X$.

Proof. Suppose first that $R$ is a complete $\boldsymbol{\Sigma}_{1}^{1}$ quasi-order on $X$. If $F$ is a $\boldsymbol{\Sigma}_{1}^{1}$ equivalence relation on the Polish space $Y, F$ is in particular a quasi-order, hence Borel reduces to $R$. But then $F=\equiv_{F}$ Borel reduces to $\equiv_{R}$. This proves that $\equiv_{R}$ is a complete $\boldsymbol{\Sigma}_{1}^{1}$ equivalence relation.

Conversely, suppose $E$ is complete, and let $R_{0}$ be a complete $\boldsymbol{\Sigma}_{1}^{1}$ quasi-order, on say $2^{\omega}$, by Proposition 1.3. Then let $f: 2^{\omega} \rightarrow X$ be a Borel map reducing $\equiv_{R_{0}}$ to E. Set

$$
x R y \leftrightarrow x E y \vee \exists \alpha \in 2^{\omega} \exists \beta \in 2^{\omega}\left(x E f(\alpha) \wedge y E f(\beta) \wedge \alpha R_{0} \beta\right) .
$$

We claim that $R$ works. It is clearly $\boldsymbol{\Sigma}_{1}^{1}$, with $E \subseteq R$. Let

$$
X_{0}=\{x \in X \exists \alpha x=f(\alpha)\}
$$

and $X_{1}=X \backslash X_{0}$. Note that for $x, y \in X_{0}$ with $x R \backslash E y$ and any $\alpha$ and $\beta$ with $x E f(\alpha)$ and $y E f(\beta)$, one must have $\alpha R_{0} \beta$, for $f$ reduces $\equiv_{R_{0}}$ to $E$. Also points in $X_{0}$ and $X_{1}$ are $R$-unrelated, and $\left.R\right|_{X_{1}}=\left.E\right|_{X_{1}}$. This easily implies that $R$ is 
indeed a quasi-order, that $\equiv_{R}=E$, and that $f$ is a reduction of $R_{0}$ to $R$, so that $R$ is complete.

In the applications, we will only use the "if" part, proving first that certain $\Sigma_{1}^{1}$ quasi-orders are complete, and derive that the associated equivalence relation is complete too. But the "only if" part indicates that complete $\boldsymbol{\Sigma}_{1}^{1}$ equivalence relations are always of this form (although the quasi-ordering might not be as "natural" as the equivalence relation). This is some indication that the limitations of our method might be intrinsic.

To end this introductory section, let us briefly discuss some of the milestones discovered in the theory of $\boldsymbol{\Sigma}_{1}^{1}$ equivalence relations that will be useful later to put the results of sections 3 and 4 in perspective. References can be found in Becker-Kechris [2, Hjorth [12] and Kechris [13, 14, 15].

Given a Polish group $G$ and a Borel action $a$ of $G$ on some Polish space $X$, the orbit equivalence relation $E_{G}^{X}$ is defined, on $X$, by

$$
x E_{G}^{X} y \leftrightarrow \exists g \in G a(g, x)=y .
$$

Call such an $E_{G}^{X}$ a $G$-equivalence relation (it is always $\Sigma_{1}^{1}$ ). Then the class of all $G$-equivalence relations admits a complete element (see Becker-Kechris [2]), usually denoted by $E_{G}^{\infty}$, which we call the $G$-complete equivalence relation. Here are some examples:

(i) $\left(2^{\omega},=\right)$ is $G$-complete for all compact groups $G$, and also for all $\Pi_{1}^{0}$ (and even $\Pi_{2}^{0}$ ) equivalence relations.

(ii) The relation $E_{0}$ on $2^{\omega}$, defined by

$$
\alpha E_{0} \beta \leftrightarrow\{n: \alpha(n) \neq \beta(n)\} \text { is finite }
$$

is $\mathbb{Z}$-complete.

(iii) $E_{F_{2}}^{\infty}$, where $F_{2}$ is the free group with 2 generators, is complete for the class of all $G$-equivalences, $G$ a Polish locally compact group.

(iv) Graph isomorphism (i.e. isomorphism between countable graphs with domain $\mathbb{N}$ ) is $S_{\infty}$-complete, where $S_{\infty}$ is the symmetric group (of permutations of $\mathbb{N})$.

(v) If $G_{0}$ is either the group of homeomorphisms of the Hilbert cube, or the group of isometries of the Urysohn space (see section 3), $E_{G_{0}}^{\infty}$ is complete for the class of all $\boldsymbol{\Sigma}_{1}^{1}$ equivalence relations which are $G$-equivalence relations for some Polish group $G$.

(vi) Not every $\Sigma_{1}^{1}$ equivalence relation is Borel reducible to a $G$-equivalence relation for some Polish group $G$. The simplest counterexample is $E_{1}$, defined on $\left(2^{\omega}\right)^{\omega}$ by

$$
\left(\alpha_{n}\right) E_{1}\left(\beta_{n}\right) \leftrightarrow\left\{n: \alpha_{n} \neq \beta_{n}\right\} \text { is finite. }
$$

(vii) From the previous discussion, we also have complete equivalence relations $E_{\boldsymbol{\Sigma}_{1}^{1}}$ and $E_{\mathbf{K}_{\sigma}}$ in the classes of $\boldsymbol{\Sigma}_{1}^{1}$ and $\mathbf{K}_{\sigma}$ equivalence relations, respectively.

(viii) The relation $E_{\text {cntble }}$ is defined on $\mathbb{R}^{\omega}$ by

$$
\left(\alpha_{n}\right) E_{\text {cntble }}\left(\beta_{n}\right) \leftrightarrow\left\{\alpha_{n}: n \in \omega\right\}=\left\{\beta_{n}: n \in \omega\right\} .
$$

The order $\leq_{B}$, between these milestones, is given by:

a) $(\omega,=)<_{B}\left(2^{\omega},=\right)<_{B} E_{0}<_{B} E_{1}<_{B} E_{\mathbf{K}_{\sigma}}<_{B} E_{\boldsymbol{\Sigma}_{1}^{1}}$,

b) $E_{0}<_{B} E_{F_{2}}^{\infty}<_{B} E_{\mathbf{K}_{\sigma}}$,

c) $E_{F_{2}}^{\infty}<_{B} E_{\text {cntble }}<_{B} E_{S^{\infty}}^{\infty}<_{B} E_{G_{0}}^{\infty}<_{B} E_{\Sigma_{1}^{1}}$, 
d) the pairs $\left(E_{1}, E_{G_{0}}^{\infty}\right)$ and $\left(E_{\text {cntble }}, E_{\mathbf{K}_{\sigma}}\right)$ are $\leq_{B}$-incomparable.

One should add that this picture does not reflect at all the complexity of $\leq_{B}$. For example, the interval $\left[E_{0}, E_{F_{2}}^{\infty}\right]$ is already immensely complicated. Also, there are $\boldsymbol{\Sigma}_{1}^{1}$ equivalence relations "on the side", even at the $(\omega,=)$ level, i.e. $\boldsymbol{\Sigma}_{1}^{1}$ equivalence relations having $\aleph_{1}$ classes but no perfect set of inequivalent elements. For more details, we refer the reader to the above-mentioned books.

\section{The BASIC EXAMPLE $\leq_{\max }$}

Our aim is to define a "combinatorially simple" complete $\boldsymbol{\Sigma}_{1}^{1}$ quasi-order. But let us first discuss two simpler cases, the compact and $\mathbf{K}_{\sigma}$ quasi-orders.

Suppose first that $R$ is a compact quasi-order, on some compact Polish space $X$. To each $x \in X$, we can associate the compact set $R_{x}=\{y \in X: y R x\}$, and clearly

$$
x R y \leftrightarrow R_{x} \subseteq R_{y} .
$$

So the map $x \mapsto R_{x}$ from $X$ into the space $\mathcal{K}(X)$ of compact subsets of $X$ is a reduction of $R$ to $\subseteq$. As a consequence, we get:

Proposition 2.1. $(\mathcal{P}(\omega), \subseteq)$ is a complete compact quasi-order.

Proof. Given $(X, R)$ as above, choose an open basis $\left(U_{n}\right)_{n \in \omega}$ of $X$, and set $f(x)=$ $\left\{n: U_{n} \cap R_{x} \neq \emptyset\right\}$. By the previous discussion, $f$ is a reduction of $R$ to $\subseteq$, and $f$ is Borel (this is the point that does not work for $\Pi_{1}^{0}$ quasi-orders. It is still true that $(\mathcal{P}(\omega), \subseteq)$ is complete for them, but some work has to be done).

If we want to do the same thing for a $\mathbf{K}_{\sigma}$ quasi-order $R$, there is a problem. The reduction map $x \mapsto R_{x}$ is still Borel (in the codes), but inclusion between $\mathbf{K}_{\sigma}$ sets is $\boldsymbol{\Pi}_{1}^{1}$ in the codes, but not $\mathbf{K}_{\sigma}$ (not even $\boldsymbol{\Sigma}_{1}^{1}$ ). The idea is to replace inclusion by a stronger relation, taking advantage of the transitivity of $R$.

Let $\leq_{\mathbf{K}_{\sigma}}$ be the quasi-order on $\mathcal{P}(\omega)^{\omega}$ defined by

$$
\left(A_{n}\right) \leq_{\mathbf{K}_{\sigma}}\left(B_{n}\right) \leftrightarrow \exists n \forall m A_{m} \subseteq B_{m+n} .
$$

Proposition 2.2. $\leq_{\mathbf{K}_{\sigma}}$ is complete for $\mathbf{K}_{\sigma}$ quasi-orders (on compact Polish spaces).

Proof. Let $R$ be a $\mathbf{K}_{\sigma}$ quasi-order on the compact Polish space $X$. Write $R=$ $\bigcup_{n} R^{n}$, where the sets $R^{n}$ are compact, increasing, and satisfy $R_{0}=\Delta$, the diagonal of $X$, and for all $n R^{n} \circ R^{n} \subseteq R^{n+1}$, where $R^{n} \circ R^{n}=\{(x, z): \exists y(x, y) \in$ $\left.R^{n} \wedge(y, z) \in R^{n}\right\}$. This is easy to get from an arbitrary sequence of compact sets with union $R$, as the operation o preserves compactness. Now fix an open basis $\left(U_{n}\right)$ for $X$, and define $f: x \in X \mapsto\left(A_{x}^{n}\right) \in \mathcal{P}(\omega)^{\omega}$, by setting $A_{x}^{n}=\left\{m: U_{m} \cap R_{x}^{n} \neq \emptyset\right\}$. Clearly $f$ is Borel, so we have to check that it reduces $R$ to $\leq_{\mathbf{K}_{\sigma}}$. If $f(x) \leq_{\mathbf{K}_{\sigma}} f(y)$, then in particular $R_{x} \subseteq R_{y}$ and $x R y$. Conversely if $x R y$, then for some $n x R^{n} y$. But then for $m \geq n, z \in R_{x}^{m}$ implies $z \in R_{y}^{m+1}$. So for all $m R_{x}^{m} \subseteq R_{y}^{m+n+1}$, hence $n+1$ witnesses that $f(x) \leq_{\mathbf{K}_{\sigma}} f(y)$.

By Proposition 1.5, the associated equivalence relation $\equiv_{\mathbf{K}_{\sigma}}$ is complete among $\mathbf{K}_{\sigma}$ equivalence relations. Another very similar $\mathbf{K}_{\sigma}$ equivalence relation was already known to be complete (see Kechris [13]).

For the general case of $\boldsymbol{\Sigma}_{1}^{1}$ quasi-orders, our plan is similar. Inclusion between $\boldsymbol{\Sigma}_{1}^{1}$ sets is now $\boldsymbol{\Pi}_{2}^{1}$ in the codes, so we must replace it by a stronger quasi-order, again taking advantage of transitivity. And, as we did above, we will change it slightly to get a more combinatorial object. 
First we need some notations and terminology.

Given a set $X, X^{<\omega}$ denotes the set of finite sequences from $X$. If $s \in X^{<\omega},|s|$ is its length (its domain), and $s \subseteq t$ means $s$ is a restriction of $t$. If $X=\omega$, we also define $s \leq t$ by $|s|=|t|$ and $\forall i<|s| s(i) \leq t(i)$. Similarly $s+t$, for $s$ and $t$ of the same length, is defined by pointwise addition. We view elements in $(X \times Y)^{<\omega}$ as pairs in $X^{<\omega} \times Y^{<\omega}$ of the same length.

A (set-theoretical) tree on $X$ is a subset of $X^{<\omega}$ closed under restrictions. If $T$ is a tree on $X \times \omega$, we say that $T$ is normal if whenever $(u, s) \in T$ and $s \leq t$, $(u, t) \in T$, too. For $s \in \omega^{<\omega}$, set

$$
T(s)=\left\{u \in X^{<\omega}:|u|=|s| \wedge(u, s) \in T\right\} .
$$

So $T$ is normal if $s \leq t$ implies $T(s) \subseteq T(t)$.

A map $f: \omega^{<\omega} \rightarrow \omega^{<\omega}$ is Lipschitz if $f$ preserves both length and extension. This is equivalent to saying that there is a map $f^{*}: \omega^{<\omega} \times \omega \rightarrow \omega$ such that $f$ is obtained by the recursion $f(\emptyset)=\emptyset$ and $f\left(s^{\frown} n\right)=f(s)^{\frown} f^{*}(s, n)$. It is also the same as saying that $f$ corresponds to a strategy of Player II in the usual type of games on $\omega$, where the two players alternate playing integers.

Definition 2.3. We let $\mathcal{T}$ be the space of normal trees on $2 \times \omega$. Topologized as a subset of $2^{(2 \times \omega)^{<\omega}}$, it is Polish. We then define $\leq_{\max }$ on $\mathcal{T}$ by

$$
S \leq_{\max } T \leftrightarrow \exists f: \omega^{<\omega} \rightarrow \omega^{<\omega} \forall s \in \omega^{<\omega} S(s) \subseteq T(f(s)) .
$$

(Going back to the discussion above, if we view a tree $T$ as coding the $\boldsymbol{\Sigma}_{1}^{1}$ subset of $2^{\omega}$,

$$
p([T])=\left\{\alpha: \exists \beta \in \omega^{\omega} \forall n\left(\left.\alpha\right|_{n},\left.\beta\right|_{n}\right) \in T\right\},
$$

then $S \leq_{\max } T$ is a strong way of saying that $p([S]) \subseteq p([T])$.)

$\leq_{\max }$ is clearly a $\boldsymbol{\Sigma}_{1}^{1}$ quasi-order on $\mathcal{T}$. In order to show it is complete, we first prove a normal form result for $\Sigma_{1}^{1}$ quasi-orders on $2^{\omega}$.

Theorem 2.4. Let $R$ be a $\boldsymbol{\Sigma}_{1}^{1}$ quasi-order on $2^{\omega}$. Then there exists a tree $S$ on $2 \times 2 \times \omega$ satisfying:

(i) $R=p([S])$, i.e. $\alpha R \beta \leftrightarrow \exists \gamma \in \omega^{\omega} \forall n\left(\left.\alpha\right|_{n},\left.\beta\right|_{n},\left.\gamma\right|_{n}\right) \in S$.

(ii) $S$ is normal, i.e. $(u, v, s) \in S$ and $s \leq t$ imply $(u, v, t) \in S$.

(iii) If $u \in 2^{<\omega}$ and $s \in \omega^{<\omega}$ are of the same length, $(u, u, s) \in S$.

(iv) If $(u, v, s) \in S$ and $(v, w, t) \in S$, then $(u, w, s+t) \in S$.

Proof. Start with any tree $T_{0}$ on $2 \times 2 \times \omega$ with $R=p\left(\left[T_{0}\right]\right)$. As is well known, if we set

$$
T_{1}=\left\{(u, v, t): \exists s \leq t(u, v, s) \in T_{0}\right\},
$$

$T_{1}$ is normal, and we still have $R=p\left(\left[T_{1}\right]\right)$. Also, if we let $T_{2}=T_{1} \cup\{(u, u, s)$ : $|u|=|s|\}, T_{2}$ now satisfies (i), (ii) and (iii) (it satisfies (i) because $R$ is reflexive).

Finally we define $S$ by $(\emptyset, \emptyset, \emptyset) \in S$, and for all $k, n \in \omega, u, v \in 2^{k}, s \in \omega^{k}$ and $i, j \in 2$,

$$
\begin{aligned}
& (u \frown i, v \frown j, n \frown s) \in S \\
& \Longleftrightarrow \exists u_{0}, u_{1}, \ldots, u_{n} \in 2^{k}\left(u_{0}=u \wedge u_{n}=v \wedge \forall l<n\left(u_{l}, u_{l+1}, s\right) \in T_{2}\right)
\end{aligned}
$$

(so if $n=0,\left(u \frown i, v \frown j, 0^{\frown} s\right) \in S$ if and only if $u=v$ ).

We claim that $S$ works. Clearly it is a tree. To check (i), note first that if $(x, y, \alpha)$ is a branch through $T_{2}$, then $(x, y, 1 \frown \alpha)$ is a branch through $S$. So $R \subseteq p([S])$. Conversely, suppose $\left(x, y, n^{\frown} \alpha\right)$ is a branch through $S$. If $n=0, x=y$, and 
$(x, y) \in R$. If $n>0$, we get, for each $k$, sequences $\left(u_{i}^{k}\right)_{i \leq n}$ in $2^{k}$, with $u_{0}^{k}=\left.x\right|_{k}$, $u_{n}^{k}=\left.y\right|_{k}$, and for $i<n,\left(u_{i}^{k}, u_{i+1}^{k},\left.\alpha\right|_{k}\right) \in T_{2}$. By compactness of $2^{\omega}$, we can find a subsequence $\left(k_{l}\right)$ and for $i \leq n$ elements $z_{i} \in 2^{\omega}$ such that $u_{i}^{k_{l}} \rightarrow z_{i}$ as $l \rightarrow \infty$. But then we get that for $i<n,\left(z_{i}, z_{i+1}, \alpha\right)$ is a branch through $T_{2}$, hence $z_{i} R z_{i+1}$. As $z_{0}=x$ and $z_{n}=y$, we get by transitivity $x R y$, as desired.

To check (ii), let $(u, v, s) \in S$ and $t \geq s$. The case of $(\emptyset, \emptyset, \emptyset)$ is trivial. So suppose $u=u^{\prime} \frown i, v=v^{\prime} j, s=n \frown s^{\prime}$ and $t=m \frown t^{\prime}$, with $n \leq m$ and $s^{\prime} \leq t^{\prime}$. As $T_{2}$ is normal, we also have $\left(u, v, n \frown t^{\prime}\right) \in S$, with the same witnesses $\left(u_{i}\right)_{i \leq n}$. Also, using property (iii) of $T_{2}$, we can first repeat $m-n$ times the witness $u_{0}$ to get witnesses for $\left(u, v, m \frown t^{\prime}\right) \in S$, as desired.

(iii) follows from (ii) and the immediate remark that if $|u|=|s|$ and $s(0)=0$, $(u, u, s) \in S$.

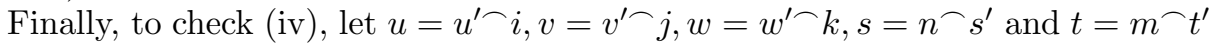
satisfy $(u, v, s) \in S$ and $(v, w, t) \in S$. By (ii), we also have

$$
\left(u, v, n \frown\left(s^{\prime}+t^{\prime}\right)\right) \in S \quad \text { and } \quad\left(v, w, m \frown\left(s^{\prime}+t^{\prime}\right)\right) \in S,
$$

as witnessed by say $\left(u_{i}\right)_{i \leq n}$ and $\left(v_{j}\right)_{j \leq m}$, respectively. But then $\left(u_{i}\right)_{i<n}\left(v_{j}\right)_{j \leq m}$ is a witness that $\left(u, w,(n+m) \frown\left(s^{\prime}+t^{\prime}\right)\right) \in S$, as desired.

As a consequence, we get:

Theorem 2.5. The quasi-order $\leq_{\max }$ is a complete $\boldsymbol{\Sigma}_{1}^{1}$ quasi-order.

Proof. We only need to Borel reduce to $\leq_{\max }$ any $\boldsymbol{\Sigma}_{1}^{1}$ quasi-order $R$ on $2^{\omega}$. Then let $S$ be the tree associated to $R$ by Theorem 2.4 , and define $f: 2^{\omega} \rightarrow \mathcal{T}$ by

$$
f(x)=S^{x}=\left\{(u, s) \in(2 \times \omega)^{<\omega}:\left(u,\left.x\right|_{|u|}, s\right) \in S\right\} .
$$

Indeed the tree $S^{x}$ is normal, as $S$ is. And $f$ is Borel, in fact continuous. We check that it is a reduction. Suppose first that $S^{x} \leq_{\max } S^{y}$, as witnessed by the Lipschitz map $\varphi: \omega^{<\omega} \rightarrow \omega^{<\omega}$. If $0^{k}$ is the sequence of length $k$ with constant value 0 , the sequences $\varphi\left(0^{k}\right), k \in \omega$, extend each other, hence build $\alpha \in \omega^{\omega}$. By property (iii), for all $k,\left(\left.x\right|_{k}, 0^{k}\right) \in S^{x}$, hence $\left(\left.x\right|_{k}, \varphi\left(0^{k}\right)\right) \in S^{y}$. So $(x, y, \alpha)$ is a branch through $S$ and by (i) $x R y$.

Conversely, suppose $x R y$, and let $\alpha$ be such that $(x, y, \alpha)$ is a branch through $S$. Define $\varphi: \omega^{<\omega} \rightarrow \omega^{<\omega}$ by $\varphi(s)=s+\left.\alpha\right|_{|s|}$. The map $\varphi$ is clearly Lipschitz. Also, if $s \in 2^{k}$, and $u$ is such that $(u, s) \in S^{x}$, we get $\left(u,\left.x\right|_{k}, s\right) \in S$, and $\left(\left.x\right|_{k},\left.y\right|_{k},\left.\alpha\right|_{k}\right) \in S$; hence by property (iv) of $S,\left(u,\left.y\right|_{k}, \varphi(s)\right) \in S$ and $(u, \varphi(s)) \in S^{y}$. So $\varphi$ witnesses $S^{x} \leq_{\max } S^{y}$, as desired.

Remark 2.6. 1. In the previous proof, we only used a weak consequence of the fact that $S^{x} \leq_{\max } S^{y}$ in one direction, and proved a strong form of it in the other direction. So this leaves room for proving that many variants of $\leq$ max are also complete. The simplest to define is probably $\leq_{\text {max }}^{*}$, defined on $\mathcal{T}$ by

$$
S \leq_{\max }^{*} T \leftrightarrow \exists \alpha \in \omega^{\omega} \forall(u, s) \in S\left(u, s+\left.\alpha\right|_{|s|}\right) \in T,
$$

which is also more in the spirit of the $\mathbf{K}_{\sigma}$ case. But our goal is to get a complete $\Sigma_{1}^{1}$ quasi-order which is easy to reduce to other quasi-orders, and in this respect, $\leq_{\max }$ seems more useful in practice.

2. Some of the features of $\leq_{\max }$ may look superfluous, like e.g. insisting that we consider only normal trees (of course its natural extension to arbitrary trees is 
complete, too). But note that because the trees are normal, Lipschitz witnesses to $S \leq{ }_{\max } T$ can always be chosen one-to-one, a fact we will use later on.

Also, one can view the trees $T$ as labelings of the basic tree $\omega^{<\omega}$ by finite subsets of a countable set (associating to $s \in \omega^{<\omega}$ the labeling $T(s)$ ), and naturally extend $\leq_{\max }$ to more general labelings, by finite or even infinite subsets of say $\omega$, and again obtain complete $\boldsymbol{\Sigma}_{1}^{1}$ quasi-orders. But again, we will later use the specific labeling given by the elements of $\mathcal{T}$.

3. The ordering $\leq_{\max }$ also admits a game-theoretic interpretation. For $S, T$ in $\mathcal{T}$, consider the game $G(S, T)$ where two players alternate playing integers, Player I building a sequence $\alpha$ and Player II a sequence $\beta$, and where Player II wins the run if for all $n, S\left(\left.\alpha\right|_{n}\right) \subseteq T\left(\left.\beta\right|_{n}\right)$. Then clearly $S \leq_{\max } T$ if and only if Player II has a winning strategy in $G(S, T)$.

Now $G(S, T)$ is open, uniformly in $S, T$. So by the usual analysis of open games, we get that $\neg\left(S \leq_{\max } T\right)$ is equivalent to the existence of an ordinal $\xi<\aleph_{1}$ such that Player I has a winning strategy in the following game $G^{\xi}(S, T)$ : Alternately Player I plays integers and strictly decreasing ordinals below $\xi$ and Player II answers with integers. The game finishes when the ordinal 0 is reached, the players having produced finite sequences $s$ and $t$, and Player II wins if for $i \leq|s|, S\left(\left.s\right|_{i}\right) \subseteq T\left(\left.t\right|_{i}\right)$.

Moreover the least $\xi$ for which Player I has a winning strategy in $G^{\xi}(S, T)$ provides a $\Pi_{1}^{1}$-norm on the complement of $\leq_{\max }$.

It follows that if we define $S \leq_{\max }^{\xi} T$ if Player II has a winning strategy in $G^{\xi}(S, T)$, we get an $\omega_{1}$-sequence of Borel quasi-orders which is $\leq_{B}$-cofinal in the Borel quasi-orders. For if $R$ is a Borel quasi-order, any Borel reduction $f$ of $R$ to $\leq_{\max }$ must, by a boundedness argument, also reduce $R$ to some $\leq_{\max }^{\xi}$. This line of research is pursued in a forthcoming paper by the second author (Rosendal [26]).

\section{Model-theoretic eXAMPles}

3.1. Embeddability in model theory. Given two structures $\mathcal{A}$ and $\mathcal{B}$ in the same language $L$, we say that $\mathcal{A}$ embeds into $\mathcal{B}$, in symbols $\mathcal{A} \sqsubseteq \mathcal{B}$, if there is a one-to-one map $f$ which realizes an isomorphism between $\mathcal{A}$ and the substructure $\left.\mathcal{B}\right|_{\operatorname{Im}(f)}$.

Below, we will consider only countable structures in a countable language, and will assume that the domain of the structures we consider is always $\mathbb{N}$ (so we exclude finite structures). The set $X_{L}$ of $L$-structures with domain $\mathbb{N}$ is naturally topologized as a compact zero-dimensional Polish space, and when restricted to $X_{L}$, $\sqsubseteq$ becomes a $\Sigma_{1}^{1}$ quasi-order, and the associated equivalence $\equiv$ of bi-embeddability becomes a $\Sigma_{1}^{1}$ equivalence relation.

If $\mathcal{C}$ is a class of countable $L$-structures, denote by $\sqsubseteq_{\mathcal{C}}$ and $\equiv_{\mathcal{C}}$ the restrictions of $\sqsubseteq$ and $\equiv$ to the set $X_{L} \cap \mathcal{C}$. When $\mathcal{C}$ is the set of models of a first-order theory, or of some $L_{\omega_{1} \omega}$-sentence, $X_{L} \cap \mathcal{C}$ is Borel in $X_{L}$, and $\sqsubseteq_{\mathcal{C}}$ and $\equiv_{\mathcal{C}}$ are $\boldsymbol{\Sigma}_{1}^{1}$ and fall under our study.

Historically, the first case considered was $\sqsubseteq_{L O}$, where $L O$ is the class of countable linear orders. Fraïssé [6] conjectured that $\sqsubseteq_{L O}$ is a well-quasi-order (wqo), i.e. a quasi-ordering with no infinite antichains and no infinite descending chains (or, to rephrase it in a way closer to this paper, a quasi-ordering which (Borel) reduces neither $(\omega,=)$ nor $(\omega, \geq))$. Fraïssé's conjecture was proved by Laver [16, who proved in fact that $\sqsubseteq_{L O}$ is a better-quasi-ordering (bqo), a technical strengthening of wqo due to Nash-Williams and instrumental in the proof. We will not get into 
bqo theory here (the reader may consult the nice introduction by Simpson in 23]), but just want to stress that Laver's result implies that $\sqsubseteq_{L O}$ is extremely far from complete. $\equiv_{L O}$ is also very far from complete, as it has $\aleph_{1}$ classes, but does not Borel reduce equality on $2^{\omega}$. These two facts should be contrasted to the situation of the equivalence $\cong_{L O}$ of isomorphism between countable linear orders: By a result of Friedman and Stanley (see Hjorth 12]), it is $S_{\infty}$-complete.

There have been other similar bqo results. For example, Nash-Williams 24 proves that $\sqsubseteq_{T O}$ is bqo, where $T O$ is the class of countable tree-orderings (partial orders in which the set of predecessors of a point is finite and totally ordered).

There is another notion of "tree", which we call here a combinatorial tree (to distinguish it from the set-theoretic notion of section 2, and the tree-orderings above). A combinatorial tree is a graph on some set $X$ (a symmetric antireflexive binary relation) which is connected and acyclic. A rooted combinatorial tree is a combinatorial tree together with a distinguished vertex called its root.

There are obvious relations between these different notions of "trees": If $(X, \leq)$ is a tree-ordering with a least element $x_{0}$, one gets a combinatorial tree by connecting $x$ to $y$ if one is the predecessor of the other in $\leq$. Conversely, if $\left(X, G, x_{0}\right)$ is a rooted combinatorial tree, one can define a tree-ordering on $X$ with least element $x_{0}$ by saying that $x \leq y$ if the necessarily unique path from $x_{0}$ to $y$ contains $x$. Moreover, any set-theoretical subtree $T$ of $\omega^{<\omega}$ comes with a natural tree-ordering (extension), and a natural rooted combinatorial treeing, given by $\emptyset$ and immediate extension. Up to isomorphism, we get in this way all countable tree-orderings with least element and all countable (rooted) combinatorial trees.

However, the notions of embedding for these structures are very different, as exemplified by Nash-Williams' result above and the following result:

Theorem 3.1. Let CT (respectively RCT) be the class of countable combinatorial trees (resp rooted ones). Then $\sqsubseteq_{C T}$ and $\sqsubseteq_{R C T}$ are complete $\boldsymbol{\Sigma}_{1}^{1}$ quasi-orders, and hence $\equiv_{C T}$ and $\equiv_{R C T}$ are complete $\boldsymbol{\Sigma}_{1}^{1}$ equivalence relations.

Proof. We will define a Borel reduction $T \mapsto G_{T}$ of $\leq_{\max }$ to $\sqsubseteq_{C T}$ and $\sqsubseteq_{R C T}$ (simultaneously). First, fix some one-to-one enumeration $\theta$ of $2^{<\omega}$ such that $|s| \leq$ $|t|$ implies $\theta(s) \leq \theta(t)$ (so $\theta(\emptyset)=0$ ), e.g. by using the lexicographic ordering. We now describe the combinatorial tree $G_{T}$, for $T \in \mathcal{T}$. Its domain is not $\mathbb{N}$, but it is easy to find a Borel in $T$ bijection between its domain and $\mathbb{N}$ to get the reduction we want.

First, we "double" the set $\omega^{<\omega}$, i.e. add, for each $s \in \omega^{<\omega} \backslash\{\emptyset\}$, another vertex $s^{*}$, and put an edge between $s^{*}$ and $s$, and between $s^{*}$ and the predecessor $s^{-}$of $s$. This defines a graph $G_{0}$ (not depending on $T$ ), which is clearly a combinatorial tree. Then for each pair $(u, s) \in T$, we add vertices $(u, s, x)$, where $x$ is either $0^{k}$ or

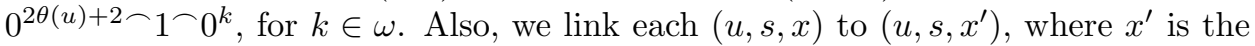
predecessor of $x$ (as a sequence), and link $(u, s, \emptyset)$ to $s$. This completely describes $G_{T}$, which is obviously a combinatorial tree. Let us make easy observations about it. First, one can compute the valence $v_{T}$ (the number of neighbors) of vertices in $G_{T}$ : elements in $\omega^{<\omega}$ have valence $\omega$, elements $\left(u, s, 0^{2 \theta(u)+2}\right)$, for $(u, s) \in T$, have valence 3 , and all other vertices have valence 2 . Next consider the distance $d_{T}$ between vertices (the length of the unique path joining them). Then (because of the doubling) the distance between vertices in $\omega^{<\omega}$ is even. The distance between a vertex $\left(u, s, 20^{\theta(u)+2}\right)$ and points in $\omega^{<\omega}$ is odd, and at least $2 \theta(u)+3$ (obtained at $s$ ). 
We now check that

$$
S \leq_{\max } T \rightarrow\left(G_{S}, \emptyset\right) \sqsubseteq\left(G_{T}, \emptyset\right)
$$

and

$$
G_{S} \sqsubseteq G_{T} \rightarrow S \leq_{\max } T,
$$

which will finish the proof.

First, suppose $S \leq_{\max } T$. Then, by the remark following Theorem 2.5, there is a one-to-one Lipschitz map $f$ from $\omega^{<\omega}$ into $\omega^{<\omega}$ with $s(s) \subseteq T(f(s))$ for $s \in \omega^{<\omega}$. Define an embedding of $G_{S}$ into $G_{T}$ as follows. Send $s \in \omega^{<\omega}$ to $f(s)$ (so in particular roots are preserved), and $s^{*}$ to $f(s)^{*}$. This defines an embedding of $G_{0}$ into itself. Next if $(u, s) \in S$, we have $(u, f(s)) \in T$, hence we can send $(u, s, x)$ to $(u, f(s), x)$. Clearly this map witnesses $\left(G_{S}, \emptyset\right) \sqsubseteq\left(G_{T}, \emptyset\right)$.

Suppose now $G_{S} \sqsubseteq G_{T}$, via some embedding $g$. Then we must have $v_{T}(g(y)) \geq$ $v_{S}(y)$ and $d_{T}(g(y), g(z))=d_{S}(y, z)$ for all vertices $y, z$ in the domain of $G_{S}$. By the remarks above, it implies that $g$ must send elements in $\omega^{<\omega}$ to elements in $\omega^{<\omega}$, i.e. defines a map $f: \omega^{<\omega} \rightarrow \omega^{<\omega}$. We claim that $f$ is a witness to $S \leq_{\max } T$.

First we show $f(\emptyset)=\emptyset$. Consider $x=\left(\emptyset, \emptyset, 0^{2}\right)$. It is a vertex in $G_{S}(S$ is infinite, hence non-empty) of valence 3 , and $d_{S}$-distance 3 from $\emptyset$. So it must be sent to some vertex of valence at least 3 in $G_{T}$, with $d_{T}$-distance 3 from $f(\emptyset)$. But by the remarks above, there is only one possible such vertex, namely $\left(\emptyset, \emptyset, 0^{2}\right)$, as points in $\omega^{<\omega}$ are at even distance of $f(\emptyset)$ and the other vertices of valence 3 are at a bigger distance. This implies in particular that $f(\emptyset)=\emptyset$.

Next we show that $f$ is Lipschitz, by induction on the length of $s$. The first step was done above. As $s^{\frown} n$ is within distance 2 from $s$ in $G_{S}, f\left(s^{\frown} n\right)$ must be within distance 2 of $f(s)$ in $G_{T}$; it cannot be $f(s)^{-}$which is $f\left(s^{-}\right)$by the induction hypothesis. So it is $f(s)-k$ for some $k$, and $f$ is Lipschitz.

Finally suppose $(u, s) \in S$, towards showing $(u, f(s)) \in T$. Consider the vertex $x=\left(u, s, 0^{2 \theta(u)+2}\right)$ in $G_{S}$. It must be sent by $g$ to some vertex $y$ in $G_{T}$ of valence at least 3 and at distance $2 \theta(u)+3$ of $f(s)$. Again points in $\omega^{<\omega}$ are forbidden by parity, so $y=\left(v, t, 0^{2 \theta(v)+2}\right)$ for some $(v, t) \in T$. But as the path in $G_{S}$ joining $s$ to $x$ does not contain $s^{-}$, the path in $G_{T}$ joining $f(s)$ to $y$ does not contain $f\left(s^{-}\right)=f(s)^{-}$, and $t$ must extend $f(s)$. But if it extends it strictly, we get $|v|>|u|$ and $\theta(v)>\theta(u)$, so that the distance is too big. So $t=f(s)$ and $\theta(v)=\theta(u)$, hence $v=u$ and finally $(u, f(s)) \in T$, as desired.

The use of points of valence $\omega$ was clearly crucial in the previous proof, and one can ask whether there is a variant of the construction that would only use locally finite combinatorial trees, i.e. trees on which the valence is finite. The next result shows the answer is negative:

Proposition 3.2. Let LFCT be the class of locally finite combinatorial trees, and let RLFCT be the class of rooted ones. Then:

(a) $R_{R L C T}$ is complete for compact quasi-orders.

(b) $\sqsubseteq_{L F C T}$ is complete for $\mathbf{K}_{\sigma}$ quasi-orders.

Proof. (a) First enumerate in a one-to-one way all isomorphism types of finite rooted trees, and let $t(H, x)$ be the number of the isomorphism type of the finite tree $H$ with root $x$. Then associate to the locally finite rooted tree $(G, x)$ the set

$$
A_{G, x}=\{t(H, x): H \text { a finite subtree of } G \wedge x \in H\} .
$$


This is clearly a Borel assignment, and we claim that it reduces $\sqsubseteq_{R L F C T}$ to $(\mathcal{P}(\omega)$, $\subseteq)$. If $f$ is an embedding of $\left(G_{1}, x_{1}\right)$ into $\left(G_{2}, x_{2}\right)$ and $n \in A_{G_{1}, x_{1}}$, there is a finite subtree $H$ of $G_{1}$ with $x_{1} \in H$ and $n=t\left(H, x_{1}\right)$. But then $f(H)$ is a finite subtree of $G_{2}$ with $x_{2} \in f(H)$, and $n=t\left(f(H), x_{2}\right) \in A_{G_{2}, x_{2}}$. Conversely, suppose $A_{G_{1}, x_{1}} \subseteq A_{G_{2}, x_{2}}$. Let $G_{1}^{n}=\left\{x \in G_{1}: d_{G_{1}}\left(x_{1}, x\right) \leq n\right\}$. By local finiteness, $G_{1}^{n}$ is finite and $t\left(G_{1}^{n}, x_{1}\right)$ is in $A_{G_{1}, x_{1}}$, hence also in $A_{G_{2}, x_{2}}$. So for each $n$ there is an embedding from $\left(G_{1}^{n}, x_{1}\right)$ into $\left(G_{2}, x_{2}\right)$. But there are only finitely many possible such embeddings, and by König's lemma there is an embedding of $\left(G_{1}, x_{1}\right)$ into $\left(G_{2}, x_{2}\right)$.

We now prove the converse. For $A \subseteq \omega$, define a (rooted, locally finite) combinatorial tree $G_{A}$ as follows. Its domain is $D_{A}=\omega \times\{0\} \cup A \times\{1\}$, its root $(0,0)$ and its edges link $(i, 0)$ to $(i+1,0)$ for $i \in \omega$ and $(i, 0)$ to $(i, 1)$ for $i \in A$. Then trivially

$$
A \subseteq B \leftrightarrow\left(G_{A},(0,0)\right) \sqsubseteq\left(G_{B},(0,0)\right) .
$$

As in $G_{A}$ the valence is always $\leq 3$, and the vertices of valence 3 are the $(i, 0)$ 's, $i \in A$. This finishes the proof of (a).

(b) First we show that $\sqsubseteq_{L F C T}$ is Borel reducible to $\leq_{\mathbf{K}_{\sigma}}$. For this, associate with each locally finite combinatorial tree $G$ with domain $\mathbb{N}$ and with each integer $n$ the set

$$
A_{G}^{n}=\left\{t(H, k): H \text { is a finite subtree of } G \wedge k \in H \wedge d_{G}(0, k) \leq n\right\} .
$$

We claim that $G \mapsto\left(A_{G}^{n}\right)_{n \in \omega}$ is the (clearly Borel) reduction we want.

First suppose $f: \mathbb{N} \rightarrow \mathbb{N}$ embeds $G_{1}$ into $G_{2}$, and let $p=d_{G_{2}}(0, f(0))$. Let $m \in A_{G_{1}}^{n}$, towards showing that $m \in A_{G_{2}}^{n+p}$. Now $m=t(H, k)$ for some finite subtree $H$ of $G_{1}$ and some $k \in H$ with $d_{G_{1}}(0, k) \leq n$. So $m=t(f(H), f(k))$ and $f(H)$ is a finite subtree of $G_{2}, f(k) \in f(H)$ and $d_{G_{2}}(0, f(k)) \leq d_{G_{2}}(f(0), f(k))+p \leq n+p$, as desired.

Conversely, suppose $p$ is such that for all $n, A_{G_{1}}^{n} \subseteq A_{G_{2}}^{n+p}$. In particular (with the notations of part (a)) $A_{G_{1}}^{0}=A_{G_{1}, 0}$ is contained in $A_{G_{2}}^{p}$, which is the finite union of the $A_{G_{2}, k}$ 's for $k \in G_{2}^{p}$. But then the proof in (a) works as well to show that $G_{1}$ embeds in $G_{2}$ (with 0 sent to some $k \in G_{2}^{p}$ ).

It remains to find a Borel reduction of $\leq_{\mathbf{K}_{\sigma}}$ to $\sqsubseteq_{L F C T}$. For $\bar{A}=\left(A^{n}\right)$ a sequence of subsets of $\omega$, define a locally finite combinatorial tree $G(\bar{A})$ as follows: With the notations of part (a), its domain consists of $\{0\} \times D_{\omega \backslash\{0\}}$, on which we copy $G_{\omega \backslash\{0\}}$ and the union of the $\{n+1\} \times D_{A^{n}}$, on which we copy $G_{A^{n}}$; and we also link $(n+1,0,0)$ to $(0, n+1,0)$ for $n \in \omega$.

Suppose now $\bar{A} \leq_{\mathbf{K}_{\sigma}} \bar{B}$, so that for some $p, A^{n} \subseteq B^{n+p}$ for all $n$. Then let $f$ send $(0, n, i)$ to $(0, n+p, i)$, and $(n+1, x)$ for $x$ in $D_{A^{n}}$ to $(n+p+1, x)$. Easily $f$ embeds $G_{\bar{A}}$ into $G_{\bar{B}}$.

Conversely, suppose $f$ embeds $G_{\bar{A}}$ into $G_{\bar{B}}$. In $G_{\bar{A}}$ and $G_{\bar{B}}$, all points have valence at most 4 , and the points of valence 4 are the $(0, n+1,0)$ 's, for $n \in$ $\omega$. So in particular for some $p, f((0,1,0))=(0, p+1,0)$, and then necessarily $f((0, n+1,0))=(0, p+n+1,0)$ for all $n$ (one cannot go backwards from $(0, p+1,0)$, as there are not enough points of valence 4 in that direction). But then for each $n$, $f$ must embed the copy of $\left(G_{A^{n}},(0,0)\right)$ into the copy of $\left(G_{B^{n+p}},(0,0)\right)$, and hence $A^{n} \subseteq B^{n+p}$, as desired.

We now come back to orderings. We have seen a case, the class of countable tree-orderings, for which embeddability is very simple. Here is an opposite result. 
Theorem 3.3. Let $P O$ be the class of countable partial orders. Then $\sqsubseteq_{P O}$ is a complete $\boldsymbol{\Sigma}_{1}^{1}$ quasi-order, and hence $\equiv_{P O}$ is a complete $\boldsymbol{\Sigma}_{1}^{1}$ equivalence relation.

Proof. We will Borel reduce $\sqsubseteq_{C T}$ to $\sqsubseteq_{P O}$. This is enough by Theorem 3.1.

For each combinatorial tree $G$ on $\mathbb{N}$, we define a partial order $\leq_{G}$ as follows: Its domain is $\mathbb{N} \cup G^{*}$, where $G^{*}=\{\{n, m\}:(n, m) \in G\}$ is the set of edges of $G$. Also, we set

$$
x \leq_{G} y \leftrightarrow(x=y) \vee\left(x \in \mathbb{N} \wedge y \in G^{*} \wedge x \in y\right) .
$$

Clearly each $\leq_{G}$ is a partial order (there is no transitivity to be checked), and it is easy to transform it into a partial order on $\mathbb{N}$, in a Borel way in $G$. So it remains to check that it gives a reduction.

First if $f$ is an embedding of $G_{1}$ into $G_{2}, f$ extends to $G_{1}^{*}$ by sending $\{n, m\}$ to $\{f(n), f(m)\}$, and this gives an embedding of $\leq_{G_{1}}$ into $\leq_{G_{2}}$.

Conversely, if $f$ is an embedding of $\leq_{G_{1}}$ into $\leq_{G_{2}}$, as any $n \in \mathbb{N}$ is $\leq_{G_{1}}$-below at least some edge, $f$ must send $\mathbb{N}$ to $\mathbb{N}$, and similarly $f$ must send $G_{1}^{*}$ to $G_{2}^{*}$. As each edge $\left\{\{n, m\}\right.$ in $G_{1}^{*}$ is above $n$ and $m$ and nothing else, we must have $f(\{n, m\})=\{f(n), f(m)\}$. This implies that $n G_{1} m \rightarrow f(n) G_{2} f(m)$, but also that adjacent edges in $G_{1}$ must go to adjacent edges in $G_{2}$, so that finite paths are preserved, and hence distance, too. In particular if $n$ and $m$ are not $G_{1}$-related, their images cannot be $G_{2}$-related, and $f$ defines an embedding of $G_{1}$ into $G_{2}$.

Remark 3.4. 1. The partial orders above are very close to being lattices. Indeed if we add a maximum element and a minimum element to them, they become lattices. As the proof of Theorem 3.3 goes (essentially) unchanged, we get as a corollary of the proof that embeddability between countable lattices (viewed as partial orders), and embeddability between countable lattices (viewed as lattices) are complete $\boldsymbol{\Sigma}_{1}^{1}$ quasi-orders.

2. Theorem 3.3 is one manifestation of a nice "reflection" phenomenon:

The quasi-ordering $\leq_{B}$ should be considered (at least we hope) as one of the "natural" quasi-orderings occurring in mathematics. As it was defined in section 1 , it is not of the correct type to fall within our scope, and be compared (using $\leq_{B}$ !) to other quasi-orderings. But its restrictions to many classes are (usually only "in the codes") of the correct type. This is what happens in Theorem 3.3: The restriction of $\leq_{B}$ to quasi-orders with countable domain (and discrete topology) clearly corresponds to $\sqsubseteq_{P O}$. Also, Theorem 3.3 says that this restriction of $\leq_{B}$ is already complete for $\boldsymbol{\Sigma}_{1}^{1}$ quasi-orderings.

We do not know if a similar phenomenon holds at other levels. For example, $\leq_{B}$ restricted to $\boldsymbol{\Sigma}_{1}^{1}$ quasi-orders is $\boldsymbol{\Sigma}_{3}^{1}$ (in the codes of $\boldsymbol{\Sigma}_{1}^{1}$ quasi-orders). Is it a complete $\boldsymbol{\Sigma}_{\mathbf{3}}^{\mathbf{1}}$ quasi-order?

The only known result in this direction is the following result of Adams and Kechris [1]): Consider the restriction of $\leq_{B}$ to Borel equivalence relations with countable classes (i.e. below $E_{F_{2}}^{\infty}$ ). In the codes, it is a $\boldsymbol{\Sigma}_{2}^{1}$ quasi-ordering, and as proved in [1] as a set, it is complete $\boldsymbol{\Sigma}_{\mathbf{2}}^{\mathbf{1}}$. But it is not known if it is a complete $\boldsymbol{\Sigma}_{\mathbf{2}}^{\mathbf{1}}$ quasi-order, or even if it Borel reduces $\leq_{\max }$. Adams-Kechris prove in [1] that it Borel reduces inclusion between Borel sets, hence also any Borel quasi-order.

We end this section with some open problems.

The results of this section give examples of classes $\mathcal{C}$ of countable structures such that bi-embeddability $\equiv_{\mathcal{C}}$ and isomorphism $\cong_{\mathcal{C}}$ are extremely far apart. If $\mathcal{C}$ is the 
class of countable linear orders, $\equiv_{\mathcal{C}}$ has only $\aleph_{1}$ classes, whereas $\cong_{\mathcal{C}}$ is $S_{\infty}$-complete. Also, if $\mathcal{C}$ is the class of countable partial orders, $\cong_{\mathcal{C}}$ is $S_{\infty}$-complete, whereas $\equiv_{\mathcal{C}}$ is a complete $\boldsymbol{\Sigma}_{1}^{1}$ equivalence relation. The natural question here is whether one can increase these gaps:

Question 1: Is there an $L_{\omega_{1} \omega}$-elementary class $\mathcal{C}$ with $\cong_{\mathcal{C}} S_{\infty}$-complete but $\equiv_{\mathcal{C}}$ with countably many classes?

Question 2: Is there an $L_{\omega_{1} \omega}$-elementary class $\mathcal{C}$ with $\equiv_{\mathcal{C}}$ a complete $\boldsymbol{\Sigma}_{1}^{1}$ equivalence relation but $\cong_{\mathcal{C}}$ not $S_{\infty}$-complete?

In the same vein, here is another natural question:

Question 3: Is there an $L_{\omega_{1} \omega}$-elementary class $\mathcal{C}$ with $\equiv_{\mathcal{C}}$ a complete $\boldsymbol{\Sigma}_{1}^{1}$ equivalence relation but $\sqsubseteq_{\mathcal{C}}$ not a complete $\boldsymbol{\Sigma}_{1}^{1}$ quasi-order?

A negative answer to this last question would reinforce our heuristic view that the limitations to the method developed in this paper are indeed intrinsic (and a positive answer would need a different method).

3.2. Homomorphism in model theory. Here we will concentrate on a notion of model theory somewhat complementary to that of embeddability, namely homomorphism.

We will model a graph $G$ on a set $D$ as a symmetric irreflexive relation and say that $h: D \rightarrow E$ is a homomorphism between graphs $G$ on $D$ and $H$ on $E$ if for all $x, y \in D, x G y \rightarrow h(x) H h(y)$. We write $G \preceq H$ in case there is such a homomorphism. The notion of homomorphism between graphs turns out to be very useful in combinatorics. For suppose that $G \preceq H$ and $c$ is a vertex colouring of $H$; then $c \circ h$ is a vertex colouring of $G$ and the chromatic number of $G$ will therefore be less than that of $H$.

It is known that $\preceq$ when restricted to the class of finite graphs is a very complicated relation, in fact any countable partial order embeds into it, as shown by Z. Hedrlin (see 25]). Here we intend to extend this result to the class of countably infinite graphs by showing that the relation restricted to the Polish space of countably infinite graphs is a complete analytic quasi-order.

Theorem 3.5. The quasi-order of homomorphism between countable graphs is $\boldsymbol{\Sigma}_{1}^{1}$ complete.

Proof. Let $\pi: 2^{<\omega} \hookrightarrow \omega \backslash 4$ be an enumeration such that $|u|<|v| \rightarrow \pi(u)<\pi(v)$.

Define for each $(u, s) \in(2 \times \omega)^{<\omega}$ a graph $C_{u, s}=\left(V_{u, s}, E_{u, s}\right)$ by

$V_{u, s}=\left\{s=a_{0}^{u, s}, a_{1}^{u, s}, \ldots, a_{\pi(u)}^{u, s}, b_{1}^{u, s}, \ldots, b_{2 \pi(u)}^{u, s}\right\}$ and

$a_{0}^{u, s} E_{u, s} a_{1}^{u, s} E_{u, s} \ldots E_{u, s} a_{\pi(u)}^{u, s} E_{u, s} b_{1}^{u, s}$ and $b_{i}^{u, s} E_{u, s} b_{j}^{u, s}, \forall i \neq j$.

So $C_{u, s}$ contains a complete graph on $2 \pi(u)$ points and therefore if $C_{u, s} \preceq C_{v, t}$, then $\pi(u) \leq \pi(v)$. Furthermore if such a homomorphism sends $s=a_{0}^{u, s}$ to $t=a_{0}^{v, t}$, then $\pi(u)=\pi(v)$, i.e. $u=v$.

For any $s \in \omega^{<\omega}$ let $K_{s}=\left(V_{s}, E_{s}\right)$ be a distinct copy of $K_{|s|+1}$ (the complete graph on $|s|+1$ vertices) with one of the vertices being $s$. Finally for $T \in \mathcal{T}$ let $G_{T}=\left(V_{T}, E_{T}\right)$ be defined by

$V_{T}=\bigcup_{s \in \omega<\omega} V_{s} \cup \bigcup_{(u, s) \in T} V_{u, s}$ and

$E_{T}$ consists of the edges in $E_{s}$ for $s \in \omega^{<\omega}$, in $E_{u, s}$ for $(u, s) \in T$ and $s E_{T} s^{\frown} n$ for all $s \in \omega^{<\omega}$ and $n<\omega$.

For two vertices $x, y$ in a connected graph $H$ put $d_{H}(x, y)=n$ iff $n$ is minimal such that $\exists x=x_{0}, x_{1}, \ldots, x_{n}=y x_{0} H x_{1} H \ldots H x_{n}$. We note that for $\alpha \in \omega^{\omega}$ and $n<m$ we have $d_{G_{T}}\left(\left.\alpha\right|_{n},\left.\alpha\right|_{m}\right)=m-n$ and in fact $\left.\alpha\right|_{n},\left.\alpha\right|_{n+1}, \ldots,\left.\alpha\right|_{m}$ is the 
unique shortest path from $\left.\alpha\right|_{n}$ to $\left.\alpha\right|_{m}$ in $G_{T}$. Also note that a homomorphism is necessarily distance decreasing.

Claim: If $h: G_{S} \preceq G_{T}$ for $S, T \in \mathcal{T}$, then there are some $\alpha \in \omega^{\omega}$ and $k \geq 0$ such that $\forall^{\infty} n h\left(0^{n}\right)=\left.\alpha\right|_{k+n}$.

Since $s \in \omega^{<\omega}$ is a vertex in a complete subgraph of $G_{S}$ on $|s|+1$ vertices, so is $h(s)$. So $h(s)$ can either be a vertex in some $K_{t}$ or be $b_{i}^{u, t}$ for some $t$ and $u$ (whenever $|s| \geq 2$ ). Suppose that $h(s)=b_{i}^{u, t}$ for $|s| \geq 2$ and $(u, t) \in T$. Then as $s E_{S}(s \frown 0) E_{S}\left(s \frown 0^{2}\right) E_{S} \ldots$ is a path in $G_{S}$ of vertices belonging to complete subgraphs of increasing cardinality, so must $h(s), h(s \frown 0), h\left(s \frown 0^{2}\right), \ldots$. But the valence of $a_{\pi(u)}^{u, t}$ is 2 , so $h\left(s \frown 0^{n}\right) \neq a_{\pi(u)}^{u, t}, \forall n$ and therefore $h\left(s \frown 0^{n}\right) \in\left\{b_{1}^{u, t}, \ldots, b_{2 \pi(u)}^{u, t}\right\}$ for all $n$. But this is impossible since the $b_{i}^{u, t}$ only belong to complete subgraphs of bounded cardinality $2 \pi(u)$.

So $h(s) \in K_{p(s)}$ for all $s$ with $|s| \geq 2$ and $p(s) \in \omega^{<\omega}$ such that $|p(s)| \geq|s|$. So then $d_{G_{T}}(\emptyset, h(s)) \geq|s|$ for $|s| \geq 2$.

Put $k=\inf _{n} d_{G_{T}}\left(\emptyset, h\left(0^{n}\right)\right)-n \geq 0$. Then as $d_{G_{T}}\left(0^{n}, 0^{m}\right)=m-n$ for $n<m$ and $h$ is distance decreasing, we have that $d_{G_{T}}\left(h\left(0^{n}\right), h\left(0^{m}\right)\right) \leq m-n$. Therefore $\exists m_{0} \forall n \geq m_{0} d_{G_{T}}\left(\emptyset, h\left(0^{n}\right)\right)=n+k$ and $\forall m>n \geq m_{0} d_{G_{T}}\left(h\left(0^{n}\right), h\left(0^{m}\right)\right)=m-n$.

Note that a path from $K_{s}$ to $K_{t}(s \neq t)$ in $G_{T}$ necessarily passes through $s$ and $t$, so take $n_{0} \geq m_{0}$ such that $h\left(0^{n_{0}}\right)=t_{0}$ for some $t_{0}$ with $\left|t_{0}\right| \geq n_{0}$ and $n_{1}>n_{0}$ such that $h\left(0^{n_{1}}\right)=t_{1},\left|t_{1}\right| \geq n_{1}$. Then $d_{G_{T}}\left(t_{0}, t_{1}\right)=n_{1}-n_{0}$ and $d_{G_{T}}\left(\emptyset, h\left(0_{n}\right)\right)$ is strictly increasing for $n_{0} \leq n \leq n_{1}$, so $t_{1}=t_{0} w$ for some $w$ of length equal to $n_{1}-n_{0}$. But $\left.t_{1}\right|_{\left|t_{0}\right|},\left.t_{1}\right|_{\left|t_{0}\right|+1}, \ldots, t_{1}$ is the unique shortest path from $t_{0}$ to $t_{1}$, which $h\left(0^{n_{0}}\right), \ldots, h\left(0^{n_{1}}\right)$ has to follow, so $h\left(0^{n_{0}+n}\right)=\left.t_{1}\right|_{\left|t_{0}\right|+n}$.

This shows that $h\left(0^{n_{0}}\right) \subseteq h\left(0^{n_{0}+1}\right) \subseteq \ldots$ and that therefore $\alpha=\bigcup_{n} h\left(0^{n_{0}+n}\right)$ will do. This proves the claim.

Now choose $m_{0}$ large enough so that $\forall n \geq m_{0} h\left(0^{n}\right)=\left.\alpha\right|_{k+n}$ and so that $\forall n \geq m_{0} \forall u \in 2^{n} \pi(u)>k+n+2$. Suppose $u \in 2^{n}, n \geq m_{0}$ and $\left(u, 0^{n}\right) \in S$. Then there is a vertex $x$ in $G_{S}$ of distance $\pi(u)+1$ from $0^{n}$ which belongs to a complete subgraph on $2 \pi(u)$ vertices. So there is a corresponding vertex $y$ in $G_{T}$ of distance less than $\pi(u)+1$ from $\left.\alpha\right|_{k+n}=h\left(0^{n}\right)$ which belongs to a complete subgraph of $G_{T}$ on $2 \pi(u)$ vertices. This $y$ cannot belong to some $K_{t}$, for otherwise $|t|+1 \geq 2 \pi(u)$ and $|t| \leq k+n+\pi(u)+1$, i.e. $\pi(u) \leq k+n+2$, which is impossible. So this $y$ has to be some $b_{i}^{v, t}$, with $\pi(u) \leq \pi(v)$, i.e. $|u| \leq|v|$. But for such $v$ $d_{G_{T}}\left(\left.\alpha\right|_{k+n}, b_{i}^{v, t}\right) \leq \pi(u)+1$ iff $\pi(v) \leq \pi(u)$. So $\pi(u)=\pi(v)$ and $v=u$. But then $|t|=n$ and $\left.\alpha\right|_{k+n}=t$, i.e. $k=0$ and $(u, t)=\left(u,\left.\alpha\right|_{n}\right) \in T$.

This shows that for $\left(u, 0^{n}\right) \in S$ with $n \geq m_{0}$ we have $\left(u,\left.\alpha\right|_{n}\right) \in T$. But on the image of a canonical reduction of some $\boldsymbol{\Sigma}_{1}^{1}$ quasi-order to $\leq_{\max }$ this is enough to insure that $S \leq_{\max } T$ as is seen from the proof of the maximality of $\leq_{\max }$.

On the other hand if $S \leq_{\max } T$, then it is easily seen that $G_{S}$ is in fact isomorphic to an induced subgraph of $G_{T}$, so in particular $G_{S} \preceq G_{T}$. This shows $\leq_{\max }$ reduces to the relation of homomorphism and finishes the proof.

Remark 3.6. Let us just mention that by a trivial variation of the proofs of Proposition 3.2 (b) and Theorem 3.5 one can show that the relation of homomorphism between countable locally finite connected graphs is Borel bi-reducible with $\mathbf{K}_{\sigma}$. Note also that the above proof of course produces connected graphs, as is easily seen. 


\section{EMBEDDABILITY IN ANALYSIS}

In this section, we consider various "embeddability" notions that occur in analysis, i.e. that are defined between separable structures (as opposed to countable structures), like Polish metric spaces, compact metrizable spaces and separable Banach spaces.

4.1. Isometric embeddability. A Polish metric space $(X, d)$ is a metric space in which the distance $d$ is complete, and the induced topology is Polish (which just means separable here). The distance is very often understood.

A map $f:\left(X, d_{X}\right) \rightarrow\left(Y, d_{Y}\right)$ is an isometric embedding if it preserves the distances. It is an isometry between $X$ and $Y$ if moreover it is onto $Y$. This naturally leads to the notions of isometric embeddability $\complement^{i}$, isometric bi-embeddability $\equiv^{i}$ and isometry $\cong i$, defined respectively by

$X \sqsubseteq^{i} Y \leftrightarrow \exists f: X \rightarrow Y, f$ is an isometric embedding,

$X \equiv^{i} Y \leftrightarrow X \sqsubseteq^{i} Y \wedge Y \sqsubseteq^{i} X$

$X \cong{ }^{i} Y \leftrightarrow \exists f: X \rightarrow Y, f$ is an isometry.

Recall also that a Polish metric space $X$ is homogeneous if any point of $X$ can be sent to any other point by an isometry of $X$, and ultrahomogeneous if any isometry between two finite subsets of $X$ can be extended to an isometry of $X$. Also, $X$ is Heine-Borel if any closed bounded subset of $X$ is compact (so that in particular $X$ is locally compact).

The complexity of $\cong i$, for various classes of Polish metric spaces, has been studied in a very detailed way in a series of papers by Clemens, Gao and Kechris [4, 8, 5]. Our aim here is to study the complexity of the quasi-ordering $\sqsubseteq^{i}$ and the equivalence relation $\equiv^{i}$ for these classes of Polish metric spaces.

First, we have to put these objects into our frame. This is done in [8]: There is a $\sqsubseteq^{i}$-maximum space $U$ among Polish metric spaces, called the Urysohn space. It is characterized, up to isometry, by this maximality property and the fact that it is ultrahomogeneous. So, up to isometry, we can view any Polish metric space as a (necessarily closed) subspace of $U$. And if we equip $F(U)$, the set of closed subspaces of $U$, with the Effros Borel structure (and any Polish topology that gives it), the relations $\sqsubseteq^{i}, \equiv^{i}$ and $\cong^{i}$ become $\boldsymbol{\Sigma}_{1}^{1}$ on $F(U)$.

As we did in section 3 , if $\mathcal{C}$ is a class of Polish metric spaces, we use a subscript $\mathcal{C}$ to indicate the restrictions of $\sqsubseteq^{i}, \equiv^{i}$ and $\cong^{i}$ to $\mathcal{C} \cap F(U)$.

Our first result is an immediate consequence of Theorem 3.1.

Proposition 4.1. $\sqsubseteq^{i}$ is a complete $\boldsymbol{\Sigma}_{1}^{1}$ quasi-order, and $\equiv^{i}$ is a complete $\boldsymbol{\Sigma}_{1}^{1}$ equivalence relation. In fact the same is true of $\bigsqcup_{D}^{i}$ and $\equiv_{D}^{i}$, where $D$ is the class of discrete Polish metric spaces — and hence also for any intermediate class, like dim0 Polish metric spaces or locally compact Polish metric spaces.

Proof. Any countable combinatorial tree can be turned into a discrete Polish metric space by using the geodesic distance, and tree-embeddings then correspond exactly to isometric embeddings. So Theorem 3.1 gives the result. (To be less sloppy one should go, in a Borel way, from the trees to $F(U)$. But this is easy, e.g. by first embedding into $U$, as some space $X$, the tree $\omega^{<\omega}$ with its geodesic distance, and then redefine in a Borel way the reduction in Theorem 3.1 as taking values in $F(X)$, hence in $F(U)$.) 
This result should be contrasted with the results of Gao and Kechris concerning $\cong^{i}$ for the same classes: The complexity varies from $E_{S_{\infty}}^{\infty}$ for discrete Polish metric spaces, to $E_{G_{0}}^{\infty}$ for the class of all Polish metric spaces. For some intermediate classes, like locally compact Polish spaces, the exact complexity is not known.

In 8], Gao and Kechris also study isometry for the subclasses $U M$ of ultrametric Polish spaces, HUM of homogeneous ultrametric Polish spaces and $U H U M$ of ultrahomogeneous ultrametric Polish spaces. They prove that $\cong_{U M}^{i}$ is Borel bireducible with $E_{S_{\infty}}^{\infty}$, and $\cong_{H U M}^{i}$ and $\cong_{U H U M}^{i}$ are Borel bi-reducible with $E_{\text {cntble }}$ (see the end of section 1 for the definitions).

Here we have:

Proposition 4.2. (a) $\bigsqcup_{U M}^{i}$ is a complete $\boldsymbol{\Sigma}_{1}^{1}$ quasi-order, and hence $\equiv_{U M}^{i}$ is a complete $\boldsymbol{\Sigma}_{1}^{1}$ equivalence relation.

(b) $\bigsqcup_{H U M}^{i}$ and $\bigsqcup_{U H U M}^{i}$ are Borel bi-reducible with the ordering $\subseteq_{c n t b l e}$, defined on $\mathbb{R}^{\omega}$ by

$$
\left(x_{n}\right) \subseteq_{\text {cntble }}\left(y_{n}\right) \leftrightarrow\left\{x_{n}: n \in \omega\right\} \subseteq\left\{y_{n}: n \in \omega\right\} .
$$

$S o \equiv_{H U M}^{i}$ and $\equiv_{U H U M}^{i}$ are bi-reducible with $E_{\text {cntble }}$, but in fact for homogeneous ultrametric spaces, isometric bi-embeddability and isometry coincide.

Proof. (a) If $\left(G, x_{0}\right)$ is a rooted countable combinatorial tree, its body $\left[G, x_{0}\right]$ is the set of infinite paths through $G$ starting at $x_{0}$. We turn it into an ultrametric Polish space by defining the distance between distinct paths $\left(x_{0}, x_{1}, \ldots\right)$ and $\left(x_{0}, y_{1}, \ldots\right)$ as $2^{-i}$, where $i$ is least with $x_{i} \neq y_{i}$. It is easy to check that if the trees $\left(G_{1}, x_{1}\right)$ and $\left(G_{2}, x_{2}\right)$ are pruned (every point has valence at least 2 ), embeddings from $\left(G_{1}, x_{1}\right)$ into $\left(G_{2}, x_{2}\right)$ correspond exactly to isometric embeddings of their bodies.

But then note that in the proof of Theorem 3.1 we only used pruned trees, and working a bit to get the bodies as subspaces of $U$ (by e.g. embedding first the "universal" body $\left.\omega^{\omega}\right)$, we get (a).

(b) The following argument is similar to the one in 8. First recall that given a countable subset $\mathrm{A}$ of $\mathbb{R}^{+}$, there is a Polish ultrametric space $U_{A}$ which is ultrahomogeneous, has distances in $A \cup\{0\}$, and isometrically embeds all ultrametric Polish spaces with distances in $A \cup\{0\}$. So if $\theta$ is a homeomorphism between $\mathbb{R}$ and $(0,1)$ and we set $A^{*}=\{1+\theta(r): r \in A\}$ for $A$ a countable subset of $\mathbb{R}$, then the map $A \mapsto U_{A^{*}}$ gives a Borel reduction of $\subseteq_{\text {cntble }}$ to $\subseteq_{U H U M}$ (and even to its restriction to discrete spaces in $U H U M)$.

For the other direction, we have to reduce $\subseteq_{H U M}^{i}$ to $\subseteq$ cntble. So suppose $(X, d)$ is a homogeneous ultrametric Polish space. Then its set of distances $d\left[X^{2}\right]$ is countable, and for each $r \in \mathbb{R}^{+}$, the relation $d(x, y)<r$ defines on $X$ an equivalence relation $E_{r}$. Fix $x \in X$, and set, for $n \geq 2$

$$
A_{n}^{X}=\left\{r \in \mathbb{R}^{+}:\{y: d(x, y) \leq r\} \text { consists of at least } n E_{r} \text {-equivalence classes }\right\} .
$$

Note that by homogeneity, this does not depend on the choice of $x$. The sets $A_{n}^{X}$ are decreasing in $n$, and $A_{2}^{X}=d\left[X^{2}\right] \backslash\{0\}$ is countable. Our reduction is $X \mapsto A^{X}=\bigcup_{n}\left(n+\theta\left[A_{n}^{X}\right]\right)$. It is easy to see it can be enumerated in a Borel in $X$ way, and trivially if $X \sqsubseteq^{i} Y, A^{X} \subseteq A^{Y}$. Conversely suppose $A^{X} \subseteq A^{Y}$, and let $D=\left\{x_{n}: n \in \omega\right\}$ be a countable dense subset of $X$. It is enough to build an isometric embedding $f$ of $D$ into $Y$, for it can then be extended isometrically to all of $X$. We build $f$ by induction on $n$. First send $x_{0}$ to some $y_{0} \in Y$. Now suppose $f$ has been defined on $\left\{x_{0}, \ldots, x_{n}\right\}$, with $f\left(x_{i}\right)=y_{i}$. Let $r=\inf \left\{d_{X}\left(x_{n+1}, x_{i}\right), i \leq n\right\}$, $D_{r}=\left\{x_{i}: i \leq n \wedge d_{X}\left(x_{n+1}, x_{i}\right)=r\right\}$, and let $k \geq 1$ be the number of $E_{r}$-classes 
met by $D_{r}$ inside $\left\{x: d_{X}\left(x_{n+1}, x\right) \leq r\right\}$. As $x_{n+1}$ is $E_{r}$-inequivalent to all elements of $D_{r}, r \in A_{k+1}^{X}$, hence $r \in A_{k+1}^{Y}$. But this means that picking some $i$ with $x_{i}$ in $D_{r}$, there must be in $Y$ an $E_{r}^{Y}$-class inside $\left\{y: d_{Y}\left(y_{i}, y\right) \leq r\right\}$ not met by $f\left(D_{r}\right)$, hence some point $y_{n+1}$ with $d_{Y}\left(y_{n+1}, y\right)=r$ for $y \in f\left(D_{r}\right)$. Set $f\left(x_{n+1}\right)=y_{n+1}$. Defined in this way, $f$ preserves distances on $D_{r} \cup\left\{x_{n+1}\right\}$ by construction, and on all of $\left\{x_{0}, \ldots, x_{n+1}\right\}$ by the properties of ultrametrics. This finishes the proof.

For the last remark, note that the same proof, using now a back-and-forth argument, shows that for $X, Y$ in $H U M, X \equiv^{i} Y$ is equivalent to $X \cong{ }^{i} Y$ and to $A^{X}=A^{Y}$.

In [8, Gao and Kechris also consider the classes $C$ of connected locally compact Polish metric spaces, $H B$ of Heine-Borel Polish metric spaces, and $C H B$ of connected Heine-Borel Polish metric spaces. 8 8 contains a proof of Hjorth's result that isometry restricted to any of these classes is Borel-bireducible with $E_{F_{2}}^{\infty}$ (Hjorth proves in fact a stronger result, for the class of so-called pseudo-connected locally compact Polish metric spaces that contains both $C$ and $H B$; see [8]).

We do not know a good upper bound for the complexity of $\Xi_{C}^{i}$. The next result provides a lower bound, and solves the problem for $H B$ and $C H B$.

Theorem 4.3. The quasi-orders $\bigsqcup_{H B}^{i}$ and $\bigsqcup_{C H B}^{i}$ are Borel bi-reducible with $\leq_{\mathbf{K}_{\sigma}}$, and hence $\equiv_{H B}^{i}$ and $\equiv_{C H B}^{i}$ are Borel bi-reducible with $\equiv_{\mathbf{K}_{\sigma}}$.

Proof. The lower bound is easy, and follows from Proposition 3.2: Given a locally finite combinatorial tree $G$, build $X_{G}$ by replacing every edge in $G$ by a copy of $[0,1]$, and extending the geodesic distance in the natural way. The resulting space is clearly connected and Heine-Borel, and easily $G \mapsto X_{G}$ gives a Borel reduction of $\sqsubseteq_{L F C T}$ to $\Xi_{C H B}^{i}$.

To get the upper bound, we must find a Borel reduction of $\bigsqcup_{H B}^{i}$ to some $\mathbf{K}_{\sigma}$ quasi-order. This is done as follows.

We work with the Urysohn space $U$. First, for each sequence $\vec{x}=\left(x_{i}\right)_{i<n}$ in $U^{n}$, let its configuration $c(\vec{x})$ be the $n \times n$ matrix with coefficients $d\left(x_{i}, x_{j}\right)$. Let $D=\left\{(n, \vec{m}): n \in \omega, \vec{m} \in \omega^{n}\right\}$. If $\vec{K}=\left(K_{n}\right)_{n \in \omega}$ is a sequence of compact subsets of $U$ and $(n, \vec{m}) \in D$, set

$$
C_{n, \vec{m}}(\vec{K})=\left\{c\left(\vec{x}_{0} \vec{x}_{1}^{\frown \ldots} \frown \vec{x}_{n-1}\right): \forall i<n \vec{x}_{i} \in K_{i}^{m_{i}}\right\} .
$$

It is a compact subset of $\mathbb{R}^{l(\vec{m}) \times l(\vec{m})}$, where $l(\vec{m})=\sum_{i<n} m_{i}$.

We first prove:

Lemma 4.4. Suppose $\vec{K}$ and $\vec{L}$ are increasing sequences of compact subsets of $U$, with unions $X$ and $Y$, respectively. Then the following are equivalent:

(a) There is an isometric embedding $f$ from $X$ into $Y$ with $f\left(K_{n}\right) \subseteq L_{n}$ for all $n \in \omega$.

(b) For all $(n, \vec{m}) \in D, C_{n, \vec{m}}(\vec{K}) \subseteq C_{n, \vec{m}}(\vec{L})$.

Proof. (a) implies (b) is obvious, as $f$ preserves configurations. For (b) implies (a), fix a countable set $X_{0}=\left\{x_{n}: n \in \omega\right\}$ such that $X_{0} \cap K_{n}$ is dense in $K_{n}$ for each $n$. For $N \in \omega$, reenumerate $\left(x_{i}\right)_{i<N}$ by first enumerating the points in $K_{0}$, then in $K_{1} \backslash K_{0}$, and so on. This gives some $(n, \vec{m}) \in D$ and some $\vec{z}_{0}, \ldots, \vec{z}_{n-1}$ with $\vec{z}_{i} \in K^{m_{i}}$, such that the concatenation of the $z_{i}$ 's reenumerate $\left(x_{i}\right)_{i<N}$. Then $c(\vec{z})$ is in $C_{n, \vec{m}}(\vec{K})$, hence in $C_{n, \vec{m}}(\vec{L})$, and by reenumerating again, this means that there is an isometric embedding $f_{N}$ of $\left(x_{i}\right)_{i<N}$ into $Y$ satisfying $x_{i} \in K_{p} \rightarrow f\left(x_{i}\right) \in L_{p}$ 
for all $p$ and $i<N$. By compactness of the $L_{p}$ 's, one can find a subsequence of $N$ 's such that for all $i, f_{N}\left(x_{i}\right)$ converges to some $y_{i} \in L_{p(i)}$, where $p(i)$ is least with $x_{i} \in K_{p(i)}$. This defines an isometric embedding of $X_{0}$ to $Y$ which can by continuity be extended to all of $X$, and satisfies $f\left(K_{n}\right) \subseteq L_{n}$ for all $n \in \omega$.

Using this, we now finish the proof of Theorem 4.3. Fix some $x_{0} \in U$. For each Heine-Borel subspace $X$ of $U$ and $n \in \omega$, let $K_{n}(X)=\left\{x \in X: d\left(x_{0}, x\right) \leq n\right\}$, and $\vec{K}(X)=\left(K_{n}(X)\right)_{n \in \omega}$. This is an increasing sequence of compact sets, with union $X$.

Now let

$$
D^{\prime}=\{(p, q, \vec{m}): q \leq p \wedge(p-q, \vec{m}) \in D\},
$$

and define, for $(p, q, \vec{m}) \in D^{\prime}$,

$$
C_{p, q, \vec{m}}^{\prime}(X)=C_{p-q, \vec{m}}\left(T^{q} \vec{K}(X)\right),
$$

where $T^{q}(\vec{K}(i))=\vec{K}(i+q)$.

This defines a Borel map $f$ from the (Polish) space $H B$ to the compact Polish space

$$
Z=\prod_{(p, q, \vec{m}) \in D^{\prime}} \mathcal{K}(\overline{\mathbb{R}})^{l(\vec{m}) \times l(\vec{m})},
$$

equipped with the product of the Hausdorff topologies on the factors.

On $Z$, define a $\mathbf{K}_{\sigma}$ quasi-order $\leq_{Z}$ by

$$
\left(C_{i}^{1}\right)_{i \in D^{\prime}} \leq_{Z}\left(C_{i}^{2}\right)_{i \in D^{\prime}} \Longleftrightarrow \exists n \forall(p, q, \vec{m}) \in D^{\prime} C_{p, q, \vec{m}}^{1} \subseteq C_{p+n, q+n, \vec{m}}^{2} .
$$

To finish the proof, we show that $f$ reduces $\sqsubseteq_{H B}^{i}$ to $\leq_{Z}$. First, if $n$ witnesses that $\left(C_{i}^{\prime}(X)\right)_{i \in D^{\prime}} \leq_{Z}\left(C_{i}^{\prime}(Y)\right)_{i \in D^{\prime}}$, we get in particular, for $q=0$, that for any $(p, \vec{m})$ in $D, C_{p, \vec{m}}(\vec{K}(X)) \subseteq C_{p, \vec{m}}\left(T^{n} \vec{K}(Y)\right)$, and by Lemma $4.4, X$ isometrically embeds into $Y$.

Conversely, if $g$ is an isometric embedding of $X$ into $Y$, pick $x_{1}$ in $X$, and $n_{0}, n_{1}$ such that $d\left(x_{0}, x_{1}\right) \leq n_{0}$ and $d\left(x_{0}, g\left(x_{1}\right)\right) \leq n_{1}$. Then for $x$ in $K_{p}(X)$, we get $g(x)$ in $K_{p+n_{0}+n_{1}}(Y)$, and by lemma 4 again, $n_{0}+n_{1}$ witnesses that $f(X) \leq_{Z} f(Y)$, as desired.

The preceding proof is an elaboration on the proof (see Gromov [10]) that isometry for compact Polish metric spaces is tame, i.e. Borel reducible to equality on $2^{\omega}$. Analogous proofs show that $\bigsqcup^{i}$ restricted to compact Polish metric spaces, and $\sqsubseteq^{i}$ restricted to homogeneous locally compact spaces, are Borel bi-reducible with $(\mathcal{P}(\omega), \subseteq)$.

4.2. Continuous embeddability. In this subsection, we consider compact metrizable topological spaces, which we view, up to homeomorphism, as elements of the space $\mathcal{K}(I)$ of compact subsets of the Hilbert cube $I=[0,1]^{\omega}$, with its Hausdorff topology. Taking as morphisms the one-to-one continuous maps, we get the $\boldsymbol{\Sigma}_{1}^{1}$ relations of continuous embeddability $\sqsubseteq^{c}$, continuous bi-embeddability $\equiv^{c}$ and homeomorphism $\cong^{c}$ on $\mathcal{K}(I)$. As before, we indicate by a subscript $\mathcal{C}$ the restrictions of these relations to a subclass $\mathcal{C}$ of compact metrizable spaces.

The exact complexity of $\cong^{c}$ is not known. Camerlo and Gao [3] prove it is at least $E_{S_{\infty}}^{\infty}$, and Hjorth [12] proves it is strictly above it. On the other end, an easy argument (see the next subsection) shows it is at most $\cong^{i}\left(\equiv_{B} E_{G_{0}}^{\infty}\right)$. 
When restricted to 0 -dimensional spaces (i.e. $\mathcal{K}\left(2^{\omega}\right)$ ), it becomes Borel bireducible with $E_{S_{\infty}}^{\infty}[3]$. Using Cantor's analysis of countable compact sets, one easily shows that $\sqsubseteq_{\mathcal{K}([0,1])}^{c}$ is a pre-well-ordering of type $\omega_{1}+2$.

For higher dimensions, one gets:

Theorem 4.5. The quasi-order $\bigsqcup_{\mathcal{K}\left([0,1]^{2}\right)}^{c}$ is a complete $\boldsymbol{\Sigma}_{1}^{1}$ quasi-order, hence a fortiori $\sqsubseteq^{c}$, too. So, $\equiv_{\mathcal{K}\left([0,1]^{2}\right)}^{c}$ and $\equiv^{c}$ are complete $\boldsymbol{\Sigma}_{1}^{1}$ equivalence relations.

Proof. First, consider the Borel reduction $f$ of $\leq_{\max }$ to $\bigsqcup_{R C T}$ of Theorem 3.1, that we now view as taking values in the rooted set-theoretical subtrees of $\left(\omega^{<\omega}, \emptyset\right)$. Let $X$ be the image of $f$. We will use the following properties of $X$ : The elements of $X$ are pruned trees (all vertices have valence at least 2) and have $2^{\aleph_{0}}$ infinite branches, and on $X$, $\sqsubseteq_{C T}$ and $\sqsubseteq_{R C T}$ coincide. Our plan is to define a Borel map $g: \mathcal{P}\left(\omega^{<\omega}\right) \rightarrow \mathcal{K}\left([0,1]^{2}\right)$ which on $X$ reduces $\sqsubseteq_{C T}$ to $\sqsubseteq^{c}$. Composing the reductions $f$ and $g$ then gives the result.

For $A, B \in[0,1]^{2}, m(A, B)$ denotes the middle point of the segment $A B$. Given a triangle $(A, B, C)$, we define sequences $\left(A_{n}\right)_{n \leq \omega},\left(B_{n}\right)_{n<\omega},\left(A_{n}^{\prime}\right)_{n<\omega},\left(A_{n}^{\prime \prime}\right)_{n<\omega}$ and compact sets $\left(K_{n}\right)_{n<\omega}$,all depending on $(A, B, C)$, as follows:

(i) $A_{\omega}=m(A, C), A_{0}=m\left(B, A_{\omega}\right), A_{n+1}=m\left(A_{n}, A_{\omega}\right)$.

(ii) $B_{0}=m(B, C), B_{n+1}=m\left(A_{n}, C\right)$.

(iii) $A_{n}^{\prime}=m\left(A, A_{n}\right), A_{n}^{\prime \prime}=m\left(A_{n}^{\prime}, A_{n+1}^{\prime}\right)$.

(iv) $K_{n}=A A_{n} \cup A_{n} A_{n}^{\prime \prime} \cup A_{n}^{\prime \prime} A$.

(So, except for the common origin $A$, the sets $K_{n}$ are disjoint triangles which accumulate on the segment $A A_{\omega}$.)

Then define inductively, for $s \in \omega^{<\omega}$, triangles $\left(A_{s}, B_{s}, C_{s}\right)$ by

$A_{\emptyset}=(0,1), B_{\emptyset}=(0,0), C=C_{\emptyset}=(1,0)$, and

$A_{s \frown n}=A_{n}\left(A_{s}, B_{s}, C\right), B_{s \frown n}=B_{n}\left(A_{s}, B_{s}, C\right), C_{s \frown n}=C_{s}=C$.

Also let $A_{s \frown n}^{\prime}=A_{n}^{\prime}\left(A_{s}, B_{s}, C\right), A_{s \frown n}^{\prime \prime}=A_{n}^{\prime \prime}\left(A_{s}, B_{s}, C\right)$, and set $K_{\emptyset}=\emptyset$ and $K_{s \frown n}=K_{n}\left(A_{s}, B_{s}, C\right)$.

Finally, for $T \subseteq \omega^{<\omega}$, set $g(T)=K_{T}=\overline{\bigcup_{s \in T} K_{s}}$. This is clearly a Borel map, and we claim it works.

Note first that for $T \in X$, the set $K_{T}$ consists of the union of the segments $A_{s} A_{s \frown n}, A_{s} A_{s \frown n}^{\prime \prime}, A_{s \frown n}^{\prime \prime} A_{s \frown n}$ for $s^{\frown} n \in T$, of the point $C$, and of the segments $A_{s} C$ for $s \in T$ with valence $\omega$ in $T$. This is because this last set is compact containing all $K_{s}$ for $s \in T$. If $s \in T$ has infinite valence, one can pick for each $n$ with $s \frown n \in T$ an infinite branch $\alpha_{n}$ through $T$ starting by $s^{\frown} n$, and the paths $P_{n}=\bigcup_{k \geq|s|} A_{\left.\alpha_{n}\right|_{k}} A_{\left.\alpha_{n}\right|_{k+1}}$ in $K_{T}$ accumulate to $A_{s} C$.

We now check that $g$ is a reduction. First suppose that $h: S \rightarrow T$ is a rooted embedding, with $S, T$ in $X$. We then send $C$ to $C, A_{s}$ to $A_{h(s)}, A_{s}^{\prime \prime}$ to $A_{h(s)}^{\prime \prime}$ for $s \in S$, and the other points in $K_{S}$ accordingly, by linear interpolation on the corresponding segments - the only point to note here is that if $s$ has valence $\omega$ in $S$, so has $h(s)$ in $T$. This clearly defines a continuous embedding of $K_{S}$ into $K_{T}$.

Conversely, suppose $h: K_{S} \rightarrow K_{T}$ is a continuous embedding. A path in $K$ is a continuous one-to-one map $p:[0,1] \rightarrow K$. It starts at $A$ if $p(0)=A$. Two paths $p_{1}$ and $p_{2}$ starting at $A$ are essentially disjoint if for some $r \in(0,1]$, $p_{1}((0, r))$ and $p_{2}((0, r))$ are disjoint. Given $A \in K$, let $v_{K}(A)$ be the supremum of the cardinals of all families of pairwise essentially disjoint paths in $K$ starting at $A$. One immediately gets that under a continuous embedding, valence can only increase. 
Now for $S \in X$, one checks that for $s \in S, v_{K_{S}}\left(A_{s}\right)=2 . v_{S}(s)$ (with the convention $2 . \omega=\aleph_{0}$ ). Also $v_{K_{S}}(C)=2^{\aleph_{0}}$, as branches through $S$ naturally give continuous paths starting at $C$, which are essentially disjoint if the branches are distinct. Finally all other points in $K_{S}$ have valence 2 .

From this, we get that $h(C)=C$, and hence for $s \in S, h\left(A_{s}\right)=A_{k(s)}$ for some map $k: \omega^{<\omega} \rightarrow \omega^{<\omega}$. We finally check that $k$ is a tree-embedding. So let $s$ and $s \frown n$ be consecutive vertices in $S$, and consider the circuit $K_{s}{ }_{n}$ in $K_{S}$. It must be sent by $h$ to some circuit $L$ containing $A_{h(s)}$ and $A_{h(s-n)}$ but not $C$, and easily there are no such circuits in $K_{T}$ unless $h(s)$ and $h\left(s^{\frown} n\right)$ are consecutive vertices in $T$, as desired.

4.3. Separable Banach spaces. We now consider the case of separable Banach spaces. Any such space is linearly isometric to a closed subspace of $\mathcal{C}([0,1])$ equipped with the sup norm, so we can consider the Borel subset $\mathcal{B}$ of the standard Borel space of closed subsets of $\mathcal{C}([0,1])$ consisting of all closed linear subspaces of $\mathcal{C}([0,1])$, as our basic space of separable Banach spaces (equipped with some Polish topology giving its Borel structure).

Taking as morphisms the linear isometric embeddings, i.e. the linear normpreserving maps, we can define on $\mathcal{B}$ linear isometric embeddability $\sqsubseteq^{l i}$, linear isometric bi-embeddability $\equiv^{l i}$ and linear isometry $\cong^{l i}$, by

$$
\begin{aligned}
X \sqsubseteq^{l i} Y \Longleftrightarrow \exists f: X \rightarrow Y(f \text { is linear and norm-preserving }), \\
X \equiv^{l i} Y: \Longleftrightarrow X \sqsubseteq^{l i} Y \text { and } Y \sqsubseteq^{l i} X
\end{aligned}
$$

and

$$
\left.X \cong{ }^{l i} Y \Longleftrightarrow \exists f: X \rightarrow Y \text { ( } f \text { is linear, norm-preserving and onto }\right) .
$$

They are $\boldsymbol{\Sigma}_{1}^{1}$ relations on $\mathcal{B}$.

The exact complexity of the equivalence relation $\cong i$ is not known. But two classical results in Banach space theory give upper and lower bounds. First, by Banach-Stone, one has that for $K, L$ compact metrizable spaces,

$$
K \cong{ }^{c} L \leftrightarrow\left(\mathcal{C}(K),\|\|_{\infty}\right) \cong{ }^{l i}\left(\mathcal{C}(L),\|\|_{\infty}\right),
$$

and this gives a Borel reduction of $\cong^{c}$ to $\cong^{l i}$. Also, by a theorem of Mazur, any isometry between Banach spaces $X$ and $Y$ sending 0 to 0 is linear, hence on $\mathcal{B}$ the relations $\cong^{i}$ and $\cong^{l i}$ coincide (up to the codings, for one should first isometrically embed $\mathcal{C}([0,1])$ into $U$ ). So we get $\cong^{c} \leq_{B} \cong^{l i} \leq_{B} \cong^{i}$ (so that $\cong^{c} \leq_{B} \cong^{i}$ too, as stated in section 4.2). It is not known if any of these inequalities is strict.

Now consider the ordering $\sqsubseteq^{l i}$. It is not true anymore that an isometric embedding from a Banach space $X$ into a Banach space $Y$ sending 0 to 0 is necessarily linear. However, Godefroy and Kalton have very recently proved in [9] that for separable Banach spaces, $\sqsubseteq^{l i}$ still coincides with $\sqsubseteq^{i}$. So the following result can also be viewed as computing the complexity of $\sqsubseteq_{\mathcal{B}}^{i}$ and $\equiv_{\mathcal{B}}^{i}$. This result is one of a sequence of results about the complexity of various comparison notions in the theory of separable Banach spaces which are due to the second author and have appeared elsewhere [27. Most of them need a good deal of Banach space theory, and we will not discuss them here. But we could not end this paper without giving the reduction of $\sqsubseteq_{C T}$ to $\sqsubseteq^{l i}$, which was obtained by Rosendal prior to all results in this paper, and indeed was the main motivation for both developing a completeness 
method in dimension 2, and also for replacing equivalence relations by quasi-orders in this development.

Theorem 4.6. The ordering $\sqsubseteq_{C T}$ is Borel reducible to $\sqsubseteq^{l i}$, and even to its restriction to the Banach spaces isomorphic to $c_{0}$. In particular, $\square^{l i}$ is a complete $\boldsymbol{\Sigma}_{1}^{1}$ quasi-order, and $\equiv^{l i}$ a complete $\boldsymbol{\Sigma}_{1}^{1}$ equivalence relation.

Proof. Let $c_{0}$ be the usual Banach space of sequences of reals converging to 0 at infinity, with the sup-norm \|\|$_{\infty}$. We denote by $\left(e_{n}\right)_{n \in \mathbb{N}}$ the usual basis of $c_{0}$, and will write interchangeably elements of $c_{0}$ as $\left(\alpha_{n}\right)_{n \in \mathbb{N}}$ or $\sum_{n} \alpha_{n} e_{n}$.

Given a combinatorial tree $G$ with domain $\mathbb{N}$ and geodesic distance $d_{G}$, we define a new norm \|\|$_{G}$ on $c_{0}$ by

$$
\left\|\left(\alpha_{n}\right)\right\|_{G}=\sup \left\{\left|\alpha_{i}\right|+\frac{|\alpha|_{j}}{1+d_{G}(i, j)}: i, j \in \mathbb{N}, i \neq j\right\} .
$$
has

It is easy to check that \|\|$_{G}$ is indeed a norm, and that for any $\left(\alpha_{n}\right) \in c_{0}$ one

$$
\left\|\left(\alpha_{n}\right)\right\|_{\infty} \leq\left\|\left(\alpha_{n}\right)\right\|_{G} \leq \frac{3}{2}\left\|\left(\alpha_{n}\right)\right\|_{\infty},
$$

so that \|\|$_{G}$ is an equivalent norm on $c_{0}$.

Moreover, by suitably embedding isometrically $\left(c_{0},\|\|_{G}\right)$ into $\mathcal{C}([0,1])$, one can easily make the map $G \mapsto\left(c_{0},\|\|_{G}\right)$ Borel. So the proof will be finished if we can prove that this map is a reduction of $\sqsubseteq_{C T}$ to $\sqsubseteq^{l i}$, i.e. that for $G_{1}, G_{2}$ two combinatorial trees, one has

$$
G_{1} \sqsubseteq G_{2} \Longleftrightarrow\left(c_{0},\|\|_{G_{1}}\right) \sqsubseteq^{l i}\left(c_{0},\|\|_{G_{2}}\right) .
$$

One direction is easy: If $f: \mathbb{N} \rightarrow \mathbb{N}$ is an embedding of $G_{1}$ into $G_{2}, f$ must preserve the geodesic distances. So, if we define $g: c_{0} \rightarrow c_{0}$ by $g\left(\sum_{n} \alpha_{n} e_{n}\right)=$ $\sum_{n} \alpha_{n} e_{f(n)}$, we get the linear isometric embedding we wanted.

For the other direction, fix a linear isometric embedding

$$
g:\left(c_{0},\|\|_{G_{1}}\right) \rightarrow\left(c_{0},\|\|_{G_{2}}\right) .
$$

Claim. For every $p \in \mathbb{N}$, there are $i \in \mathbb{N}$ and $\epsilon \in\{-1,1\}$ such that $g\left(e_{p}\right)=\epsilon e_{i}$.

Granting this claim, the end of the proof is easy: Write $g(p)=\epsilon_{p} e_{f(p)}$, with $f: \mathbb{N} \rightarrow \mathbb{N}$. By computing norms, we get for $p \neq q$

$$
\begin{aligned}
\left\|\epsilon_{p} e_{p}+\epsilon_{q} e_{q}\right\|_{G_{1}} & =\left\|e_{f(p)}+e_{f(q)}\right\|_{G_{2}} \\
\text { and }\left\|\epsilon_{p} e_{p}+\epsilon_{q} e_{q}\right\|_{G_{1}} & =1+\frac{1}{1+d_{G_{1}}(p, q)}, \\
\text { whereas }\left\|e_{f(p)}+e_{f(q)}\right\|_{G_{2}} & =2 \text { if } f(p)=f(q), \\
\text { and }\left\|e_{f(p)}+e_{f(q)}\right\|_{G_{2}} & =1+\frac{1}{1+d_{G_{2}}(f(p), f(q))} \text { otherwise. }
\end{aligned}
$$

From these equalities, we get $f(p) \neq f(q)$ and $d_{G_{1}}(p, q)=d_{G_{2}}(f(p), f(q))$, so that $f$ embeds $G_{1}$ into $G_{2}$, as desired.

So it remains to prove the above claim. For $p \in \mathbb{N}$, set $g\left(e_{p}\right)=\sum_{i} \alpha_{i}^{p} e_{i}$. As $\left\|e_{p}\right\|_{G_{1}}=1$, we have $\frac{2}{3} \leq\left\|g\left(e_{p}\right)\right\|_{\infty} \leq 1$.

If $\left\|g\left(e_{p}\right)\right\|_{\infty}=1$, we are done, for it implies that for some $i,\left|\alpha_{i}^{p}\right|=1$, and as $\left\|g\left(e_{p}\right)\right\|_{G_{2}}=1$ too, necessarily $\alpha_{j}^{p}=0$ for $j \neq i$.

So, arguing by contradiction, suppose there is some $p$ with $\left\|g\left(e_{p}\right)\right\|_{\infty}=1-a$, for some $a$ with $0<a \leq \frac{1}{3}$. 
Consider, for $q \neq p$, the vector $e_{p}+\frac{a}{3} e_{q}$. Its $G_{1}$-norm is $1+\frac{a}{3} \cdot \frac{1}{1+d_{G_{1}}(p, q)}>1$. Now notice that the supremum, in the definition of the $G$-norms, is always attained. Applying this to the $G_{2}$-norm of $g\left(e_{p}+\frac{a}{3} e_{q}\right)$, we get that there are $i$ and $j$ (depending on $q$ ) with

$$
\left\|g\left(e_{p}+\frac{a}{3} e_{q}\right)\right\|_{G_{2}}=\left|\alpha_{i}^{p}+\frac{a}{3} \alpha_{i}^{q}\right|+\left|\alpha_{j}^{p}+\frac{a}{3} \alpha_{j}^{q}\right| \cdot \frac{1}{1+d_{G_{2}}(i, j)}>1 .
$$

From this inequality, majorizing $\left|\alpha_{i}^{q}\right|$ and $\left|\alpha_{j}^{q}\right|$ by 1 , minorizing $d_{G_{2}}(i, j)$ by 1 , and majorizing either $\left|\alpha_{i}^{p}\right|$ or $\left|\alpha_{j}^{p}\right|$ by $1-a$, we get $\left|\alpha_{i}^{p}\right|>\frac{1}{2}$ and $\left|\alpha_{j}^{p}\right|>a$. This implies in particular that when $q$ varies over $\mathbb{N}-\{p\}$, the set of corresponding pairs $(i, j)$ is finite.

It also implies that $\left|\alpha_{i}^{p}\right|>\frac{a}{3}\left|\alpha_{i}^{q}\right|$ and $\left|\alpha_{j}^{p}\right|>\frac{a}{3}\left|\alpha_{j}^{q}\right|$. So we get

$$
1+\frac{a}{3} \cdot \frac{1}{1+d_{G_{1}}(p, q)}=A_{q}+B_{q}
$$

with

$$
A_{q}=\left|\alpha_{i}^{p}\right|+\left|\alpha_{j}^{p}\right| \cdot \frac{1}{1+d_{G_{2}}(i, j)}
$$

(which depends on $q$ only via $(i, j)$ ) and

$$
B_{q}=\epsilon \cdot \frac{a}{3} \cdot\left|\alpha_{i}^{q}\right|+\epsilon^{\prime} \cdot \frac{a}{3} \cdot \frac{1}{1+d_{G_{2}}(i, j)} \cdot\left|\alpha_{j}^{q}\right|,
$$

where $\epsilon$ is the sign of $\alpha_{i}^{p} \alpha_{i}^{q}$ and $\epsilon^{\prime}$ the sign of $\alpha_{j}^{p} \alpha_{j}^{q}$.

Note also that $A_{q} \leq\left\|g\left(e_{p}\right)\right\|_{G_{2}}=1$, hence $B_{q}>0$.

Now by the remark above, we can find $q_{1} \neq q_{2}$ in $\mathbb{N}-\{p\}$ with the same $i, j, \epsilon$ and $\epsilon^{\prime}$, and say $d_{G_{1}}\left(p, q_{1}\right) \leq d_{G_{1}}\left(p, q_{2}\right)$. In particular $A_{q_{1}}=A_{q_{2}}=A$ (say). But then consider the vector $x=e_{p}+\frac{a}{3} e_{q_{1}}+\frac{a}{3} e_{q_{2}}$. Its $G_{1}$-norm is $A+B_{q_{1}}$, whereas we have, by the same computation as before,

$$
\begin{aligned}
\|g(x)\|_{G_{2}} & \geq\left|\alpha_{i}^{p}+\frac{a}{3} \alpha_{i}^{q_{1}}+\frac{a}{3} \alpha_{i}^{q_{2}}\right|+\frac{1}{1+d_{G_{2}}(i, j)} \cdot\left|\alpha_{j}^{p}+\frac{a}{3} \alpha_{j}^{q_{1}}+\frac{a}{3} \alpha_{j}^{q_{2}}\right| \\
& =A+B_{q_{1}}+B_{q_{2}},
\end{aligned}
$$

a contradiction which proves the claim and the theorem.

Remark 4.7. The construction in the previous proof also gives that for combinatorial trees $G_{1}, G_{2}$,

$$
G_{1} \cong G_{2} \leftrightarrow\left(c_{0},\|\|_{G_{1}}\right) \cong^{l i}\left(c_{0},\|\|_{G_{2}}\right),
$$

hence gives an alternative proof of $E_{S_{\infty}}^{\infty} \leq_{B} \cong l i$, which is very different from the proof based on Mazur's theorem, and gives the reduction to spaces isomorphic to $c_{0}$.

In Banach space theory, there is of course another set of natural $\boldsymbol{\Sigma}_{1}^{1}$ quasiorders and equivalence relations, associated to linear isomorphisms instead of linear isometries. Very few things are known about the complexity of these relations. It is not known in particular if isomorphism, or isomorphic bi-embeddability, between separable Banach spaces are complete $\boldsymbol{\Sigma}_{1}^{1}$ equivalence relations. The best known result in this direction is due to Rosendal [27]: The relation $E_{1}$ Borel reduces to isomorphism, so that this last relation (unlike isometry) is not Borel reducible to any $G$-equivalence, $G$ some Polish group. 


\section{QUASI-ORDERS INDUCED BY GROUPS}

In this section we will look at some quasi-order relations induced by the actions of monoids and groups. Suppose that $G$ is a Polish group acting in a Borel manner on a standard Borel space $X$. Then the induced orbit equivalence relation is analytic, but cannot be analytic complete, as even $E_{1}$ cannot reduce to it. Now if instead $G$ was not a group but only a monoid (i.e. a semigroup with an identity), then the corresponding relation on $X$, defined by $x R_{X}^{G} y \leftrightarrow \exists g \in G g \cdot x=y$, is only a quasi-order though still analytic.

Theorem 5.1. There is a Polish monoid $G$ acting continuously on a Polish space $X$, such that $R_{X}^{G}$ is a complete $\boldsymbol{\Sigma}_{1}^{1}$ quasi-order.

Proof. Let $G=S_{\infty} \times \mathcal{P}(\omega \times \omega)$ be equipped with the following product:

$$
(x, A) \cdot(y, B)=(x . y, A \cup(x \times x)[B]),
$$

where

$$
(n, m) \in(x \times x)[B] \leftrightarrow\left(x^{-1}(n), x^{-1}(m)\right) \in B .
$$

It is easily checked that this is indeed an associative product and that the element $\left(1_{S_{\infty}}, \emptyset\right)$ is a both-sided identity. Furthermore the product is continuous, so the monoid is Polish.

We let $G$ act on $\mathcal{P}(\omega \times \omega)$ by $(x, A) \cdot B=A \cup(x \times x)[B]$ and note that it is indeed associative and also clearly continuous. Moreover $\left(1_{S_{\infty}}, \emptyset\right) \cdot A=A$ for any $A \subset \omega \times \omega$. So this is a continuous action of the Polish monoid $G$ on the space $\mathcal{P}(\omega \times \omega)$, whereby $R_{\mathcal{P}(\omega \times \omega)}^{G}$ is $\boldsymbol{\Sigma}_{1}^{1}$.

We will now reduce $\sqsubseteq^{C T}$ to $R_{X}^{G}$. For $T \in \mathcal{T}$ we let $T^{\prime}=\{(2 n, 2 m) \mid(n, m) \in T\}$. Then as combinatorial trees are connected and without cycles one has for $T, S \in \mathcal{T}$

$$
T \sqsubseteq S \Leftrightarrow \exists x \in S_{\infty} n T^{\prime} m \rightarrow x(n) S^{\prime} x(m) .
$$

We now show that for $T, S \in \mathcal{T}$, we have

$$
T \sqsubseteq S \Leftrightarrow \exists g \in G g \cdot T^{\prime}=S^{\prime} .
$$

So suppose $T \sqsubseteq S$ and take $x \in S_{\infty}$ such that $n T^{\prime} m \rightarrow x(n) S^{\prime} x(m)$; then $\left(x, S^{\prime}\right) \cdot T^{\prime}=S^{\prime}$. Conversely if $(x, A) \cdot T^{\prime}=A \cup(x \times x)\left[T^{\prime}\right]=S^{\prime}$, then obviously $(x \times x)\left[T^{\prime}\right] \subset S^{\prime}$, so $n T^{\prime} m \rightarrow x(n) S^{\prime} x(m)$ and $T \sqsubseteq S$.

We will now turn to some relations induced by the translation and conjugation actions of a group on itself. For $G$ a Polish group we denote by $F(G)$ and $S g(G)$ the standard Borel spaces of the closed subsets, respectively Polish (and therefore closed) subgroups, of $G$ with the Effros Borel structure. $G$ acts on these sets by pointwise translation and conjugation, and the sets are naturally ordered by a potentially closed relation, namely that of set inclusion. But one set can also be included in another up to a translation or up to a conjugation, which are easily seen to be quasi-orders. So for $K, L \in F(G)$ let $K \subseteq{ }^{G, t} L \Leftrightarrow \exists g \in G g . K \subseteq L$ and $K \subseteq \subseteq^{G, c} L \Leftrightarrow \exists g \in G g . K . g^{-1} \subseteq L$. We write $\subseteq_{F(G)}^{G, t}$ etc. to indicate the space on which the relation is defined.

Note that if $G$ is a closed subgroup of $H$ Polish, then the inclusion mapping from $F(G)$ to $F(H)$ is a reduction of $\subseteq_{F(G)}^{G, t}$ to $\subseteq_{F(H)}^{H, t}$.

Also if $G$ is a topological factor-group of $H$, i.e. there is a continuous homomorphism of $H$ surjectively onto $G$, then $\subseteq_{F(G)}^{G, t} \leq_{B} \subseteq_{F(H)}^{H, t}$ and $\subseteq_{S g(G)}^{G, c} \leq_{B} \subseteq_{S g(H)}^{H, c}$. This is easily checked noting first that in this case the factor mapping will in fact 
be open (see 2, page 6) and that therefore the function taking the inverse image by the factor map will be Borel from $F(G)$ to $F(H)$.

Note also that if $G$ is countable, then $F(G)=\mathcal{P}(G)$.

Proposition 5.2. The quasi-orders $\subseteq_{S g\left(F_{2}\right)}^{F_{2}, c}$ and $\subseteq_{\mathcal{P}\left(\mathbb{Z}^{2}\right)}^{\mathbb{Z}^{2},}$ are $\mathbf{K}_{\sigma}$ complete.

Proof. For $\left(A_{n}\right) \in \mathcal{P}(\omega)^{\omega}$ let

$$
f\left(\left(A_{n}\right)\right)=\left\{(m, n) \mid n \in A_{m}\right\} \cup \mathbb{Z} \times\{-1\} .
$$

Then as $\mathbb{Z} \times\{-1\}$ is the only bi-infinite line in $f\left(\left(A_{n}\right)\right)$, if

$$
(m, n)+\mathbb{Z} \times\{-1\} \subseteq f\left(\left(A_{n}\right)\right),
$$

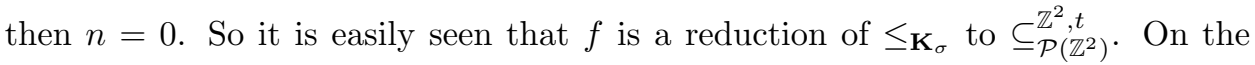
other hand, since $\mathbb{Z}^{2}$ is countable, it is easily seen that the relation $\subseteq_{\mathcal{P}\left(\mathbb{Z}^{2}\right)}^{\mathbb{Z}^{2} t}$ is indeed $\mathbf{K}_{\sigma}$.

For the other relation, we will use a construction by S. Gao in 7 to reduce $\subseteq_{\left[F_{2}\right] \omega}^{F_{2}, t}$ to $\subseteq_{S g\left(F_{2}\right)}^{F_{2}, c}$. Note that this will suffice to show that $\subseteq_{S g\left(F_{2}\right)}^{F_{2}, c}$ reduces $\leq_{\mathbf{K}_{\sigma}}$. $\mathbb{Z}^{2}$ is a topological factor-group of $F_{2}$, so the above reduction of $\leq_{\mathbf{K}_{\sigma}}$ to $\subseteq_{\mathcal{P}\left(\mathbb{Z}^{2}\right)}^{\mathbb{Z}^{2},}$ composed with the canonical reduction of $\subseteq_{\mathcal{P}\left(\mathbb{Z}^{2}\right)}^{\mathbb{Z}^{2}, t}$ to $\subseteq_{\mathcal{P}\left(F_{2}\right)}^{F_{2}, t}$ will be a reduction of $\leq_{\mathbf{K}_{\sigma}}$ to $\subseteq_{\left[F_{2}\right] \omega}^{F_{2}, t}$.

So let $F_{3}$ be the free group generated by $a, b, c$ and let $F_{2}$ be the subgroup generated by $a, b$. For an infinite subset $A$ of $F_{2}$ we let $K(A)=\left\langle x c x^{-1} \mid x \in A\right\rangle$ which is a subgroup of $F_{3}$. Then $A \mapsto K(A)$ is Borel from $\left[F_{2}\right]^{\omega}$ to $S g\left(F_{3}\right)$. Furthermore if $A, B \in\left[F_{2}\right]^{\omega}$ and $f \in F_{2}$ is such that $f . A \subseteq B$, then clearly $f . K(A) \cdot f^{-1} \subseteq K(B)$. Conversely it follows from the proof of lemma 2 in [7] that if $g \cdot K(A) \cdot g^{-1} \subseteq K(B)$ for some $g \in F_{3}$, then there is an $f \in F_{2}$ with $f . A \subseteq B$. So this shows $\subseteq_{\left[F_{2}\right] \omega}^{F_{2}, t}$ to reduce to $\subseteq_{S g\left(F_{3}\right)}^{F_{3}, c}$. But it is noted by Thomas and Velickovic in 30] that $F_{3}$ embeds as a malnormal subgroup $G_{0}$ in $F_{2}$ ( $N$ is a malnormal subgroup of $H$ iff $h . N . h^{-1} \cap N=\{1\}$ for all $\left.h \in H \backslash N\right)$. So in particular for $M, N \in S g\left(F_{3}\right)=S g\left(G_{0}\right)$ there is a $g \in F_{3}$ with $g . N . g^{-1} \subseteq M$ iff there is an $h \in F_{2}$ with h.N.h $h^{-1} \subseteq M$. So this shows the inclusion mapping from $F_{3}=G_{0}$ to $F_{2}$ to be a reduction from $\subseteq_{S g\left(F_{3}\right)}^{F_{3}, c}$ to $\subseteq_{S g\left(F_{2}\right)}^{F_{2}, c}$.

Again as $F_{2}$ is countable and $S g\left(F_{2}\right)$ is compact, $\subseteq_{S g\left(F_{2}\right)}^{F_{2}, c}$ is $\mathbf{K}_{\sigma}$.

We note that what is really shown here is that $\subseteq_{\mathcal{P}\left(\mathbb{Z}^{2}\right)}^{\mathbb{Z}, t}$ is $\mathbf{K}_{\sigma}$ complete, where $\mathbb{Z}$ is seen as the subgroup $\mathbb{Z} \times\{0\}$ of $\mathbb{Z}^{2}$. This is also more or less clear from the definition of $\leq_{\mathbf{K}_{\sigma}}$. On the other hand $\subseteq_{\mathcal{P}(\mathbb{Z})}^{\mathbb{Z}, t}$ is far from being $\mathbf{K}_{\sigma}$ complete, because the induced equivalence relation turns out to be hyperfinite, and hence $\subseteq_{\mathcal{P}(\mathbb{Z})}^{\mathbb{Z}, t}$ must be rather simple.

Proposition 5.3. The quasi-orders $\subseteq_{F\left(S_{\infty}\right)}^{S_{\infty}, t}$ and $\subseteq_{F\left(S_{\infty}\right)}^{S_{\infty}, c}$ are $\boldsymbol{\Sigma}_{1}^{1}$ complete.

Proof. Define for $T \in \mathcal{T}$ a closed subset of $S_{\infty}$ by $x \in B_{T} \leftrightarrow x(0) T^{\prime} x(1)$, where $T^{\prime}$ is defined as in the proof of Theorem 5.1. Clearly $T \mapsto B_{T}$ is Borel. So suppose that $T, S \in \mathcal{T}$ with $T \sqsubseteq S$; then there is an $x \in S_{\infty}$ such that $n T^{\prime} m \rightarrow x(n) S^{\prime} x(m)$. So if $y \in B_{T}$, then $x . y \in B_{S}$, whereby $x . B_{T} \subseteq B_{S}$. 
On the other hand if for some $x \in S_{\infty}, x . B_{T} \subseteq B_{S}$ and $n T^{\prime} m$, then for some $y \in$ $S_{\infty}$ with $y(0)=n$ and $y(1)=m, y \in B_{T}$ so $x . y \in B_{S}$ and therefore $x . y(0) S^{\prime} x . y(1)$. Thus $x(n) S^{\prime} x(m)$, which is enough to guarantee that $T \sqsubseteq S$. This shows $\sqsubseteq^{C T}$ reduces to $\subseteq_{F\left(S_{\infty}\right)}^{S_{\infty}, t}$, which finishes the proof of the first part of the proposition.

For the second part, for $n \neq m$ define $x_{m}^{n} \in S_{\infty}$ by $x_{m}^{n}(k)=k$ for $k \neq n, m$ and $x_{m}^{n}(n)=m, x_{m}^{n}(m)=n$. Then for $T \in \mathcal{T}$ let $A_{T}=\left\{x_{m}^{n} \mid n T^{\prime} m\right\} \cup\left\{1_{S_{\infty}}\right\}$, which is a countable closed subset of $S_{\infty}$. We claim that for $T, S \in \mathcal{T}$ we have $T \sqsubseteq S$ iff

$\exists y \in S_{\infty} y \cdot A_{T} \cdot y^{-1}$. To see this it is enough to note that $y \cdot x_{m}^{n} \cdot y^{-1}=x_{y(m)}^{y(n)}$, because then

$$
y \cdot A_{T} \cdot y^{-1}=\left\{x_{y(m)}^{y(n)} \mid n T^{\prime} m\right\} \cup\left\{1_{S_{\infty}}\right\} \subseteq A_{S}
$$

iff $n T^{\prime} m \rightarrow y(n) S^{\prime} y(m)$. Again this shows $\sqsubseteq^{C T}$ to reduce to $\subseteq_{F\left(S_{\infty}\right)}^{S_{\infty}, c}$, which finishes the proof.

Apart from being a somewhat natural generalization of the orbit equivalence relation of a Polish group acting on its subsets by translation and conjugation, the quasi-orders above could hopefully prove useful in the classification of the relation of embeddability between groups. In the case of isomorphism, it is known by results of Thomas and Velickovic 30] that isomorphism between finitely generated groups is a complete essentially countable equivalence relation, and it would be natural to conjecture that embedding between finitely generated groups would be $\mathbf{K}_{\sigma}$ complete as a quasi-order.

\section{REFERENCES}

1. S. Adams and A.S. Kechris, Linear Algebraic Groups and Countable Borel Equivalence Relations, J. Amer. Math. Soc. 13 (2000), 909-943. MR1775739 (2001g:03086)

2. H. Becker and A.S. Kechris, The Descriptive Set Theory of Polish Group Actions, London Math. Soc. Lecture Notes Series 232, Cambridge University Press, 1996. MR1425877 (98d:54068)

3. R. Camerlo and S. Gao, The completeness of the isomorphism relation for countable Boolean algebras, Trans. Amer. Math. Soc. 353 (2001), 491-518. MR1804507 (2001k:03097)

4. J.D. Clemens, Ph.D. thesis, U.C. Berkeley (2001).

5. J.D. Clemens, S. Gao and A.S. Kechris, Polish metric spaces: their classification and isometry groups, Bull. of Symb. Logic 7 (2001), 361-375. MR.1860610 (2002g:03099)

6. R. Fraïssé, Sur la comparaison des types d'ordre, C.R. Acad. Sci 226 (1948), 987-988 and 1330-1331.

7. S. Gao, Coding subset shift by subgroup conjugacy, Bull. London Math. Soc. 32 (2000), 653-657. MR.1781575 (2001j:03086)

8. S. Gao and A.S. Kechris, On the classification of Polish metric spaces up to isometry, Mem. Amer. Math. Soc. 161 (2003), no. 766, viii+78 pp. MR1950332 (2004b:03067)

9. G. Godefroy and N.J. Kalton, Lipschitz-free Banach Spaces, Dedicated to Professor Aleksander Pełczyński on the occasion of his 70th birthday. Studia Math. 159 (2003), no. 1, 121-141. MR2030906 (2004m:46027)

10. M. Gromov, Metric structures for Riemannian and non-Riemannian Spaces, Progress in Math. 152, Birkhaüser 1999. MR1699320 (2000d:53065)

11. G. Hjorth, Universal Co-Analytic Sets, Proc. Amer. Math. Soc. 124 (1996), 3867-3873. MR.1343698 (97b:03062)

12. G. Hjorth, Classification and orbit equivalence relations, Math. Surveys and Monographs 75 , Amer. Math. Soc., 2000. MR1725642 (2000k:03097)

13. A.S. Kechris, Lectures on definable group actions and equivalence relations, circulated notes (1994).

14. A.S. Kechris, New directions in Descriptive Set Theory, Bull. Symb. Logic 5 (1999), 161-179. MR 1791302 (2001h:03090) 
15. A.S. Kechris, Actions of Polish Groups and Classification Problems, Analysis and Logic, London Math. Soc. Lecture Notes Series, Cambridge University Press, 2000. MR 1967835 (2004b:03070)

16. R. Laver, On Fraïssé's order type conjecture, Annals of Math. 93 (1971), 89-111. MR0279005 $(43: 4731)$

17. A. Louveau, On the reducibility order between Borel equivalence relations, Stud. in Logic and Found. of Math. 134 (1994), 151-155. MR.1327979 (96g:03081)

18. A. Louveau, Two results on Borel orders, Journal of Symbolic Logic 54 (1989), 865-874. MR.1011175 (90i:03052)

19. A. Louveau, Closed quasi-orders and their vicinity, manuscript, 2001.

20. A. Louveau, Analytic partial orders, manuscript, 2002.

21. A. Louveau, C. Rosendal, Relations d'équivalence analytiques complètes, C.R. Acad. Sci. Paris 333 (2001), 903-906. MR1873805 (2002i:03054)

22. A. Louveau and B. Velickovic, A note on Borel equivalence relations, Proc. Amer. Math. Soc. 120 (1994), 255-259. MR.1169042 (94f:54076)

23. R. Mansfield and G. Weitkamp, Recursive aspects of descriptive set theory, With a chapter by Stephen Simpson. Oxford Logic Guides, 11. The Clarendon Press, Oxford University Press, New York, 1985. vii+144 pp. MR.0786122 (86g:03003)

24. C.St.J.A. Nash-Williams, On well quasi ordering infinite trees, Proc. Cambridge Phil. Soc. 61 (1965), 697-720. MR0175814 (31:90)

25. J. Nesetril, The homomorphism structure of classes of graphs, Combin. Probab. Comput. 8 (1999), no. 1-2, 177-184. MR 1684628 (2000c:05068)

26. C. Rosendal, Cofinal families of Borel quasi-orders and equivalence relations, to appear in Journal of Symbolic Logic.

27. C. Rosendal, Etude descriptive de l'isomorphisme dans la classe des espaces de Banach, Thesis, Université Paris 6 (2003).

28. S. Simpson, Bqo theory and Fraïssé's Conjecture, chapter in 23. MR0786122 (86g:03003)

29. L. Stanley, Borel diagonalization and abstract set theory: recent results of Harvey Friedman, in Harvey Friedman's research on the foundations of mathematics (L.A. Harrington et al., editors) North-Holland, Amsterdam (1985). MR0835254 (88b:03073)

30. S. Thomas and B. Velickovic, On the complexity of the isomorphism relation of finitely generated groups, J. Algebra 217 (1999), no. 1, 352-373. MR.1700491(2000i:20001)

Equipe D'Analyse Fonctionnelle, Institut de Mathématiques, Université Pierre et Marie Curie - Paris 6, Boîte 186, 4 Place Jussieu, 75252 Paris Cedex 05, France

E-mail address: louveau@ccr.jussieu.fr

Equipe D'Analyse Fonctionnelle, Institut de Mathématiques, Université Pierre et Marie Curie - Paris 6, Boîte 186, 4 Place Jussieu, 75252 Paris Cedex 05, France

Current address: Mathematics 253-37, Caltech, Pasadena, California 91125

E-mail address: rosendal@ccr.jussieu.fr 